\title{
Optical Nonlinearities of Colloidal Metal Quantum Dot - Glass Composites for Nanophotonics
}

\author{
Binita Ghosh and Purushottam Chakraborty \\ Saha Institute of Nuclear Physics, \\ India
}

\section{Introduction}

Since the inception of lasers, nonlinear optics has been a rapidly growing field of research in recent decades. It is based on the study of effects and phenomena related to the interaction of intense coherent light radiation with matter. In other words, nonlinear optics (NLO) is the branch of optics that describes the behaviour of light in nonlinear media, that is, the media in which the dielectric polarization $\mathbf{P}$ responds nonlinearly to the electric field $\mathbf{E}$ of the light. This nonlinearity is typically and only observed at very high light intensities (values of the electric field comparable to interatomic electric fields, typically $10^{8} \mathrm{~V} / \mathrm{m}$ ) such as those provided by pulsed lasers. Such high powers of laser beams made it possible, for the first time, to observe that the effect of light on a medium can indeed change its optical properties, e.g. refractive index or absorption. When this happens, the light itself also gets affected by this change in a non-linear way; for example, the non-linear response of the material can convert the laser light into new colours, both harmonics of the optical frequency and sum and difference frequencies. Typically, only a laser light is sufficiently intense to modify the optical properties of a material system. In fact, the beginning of the field of nonlinear optics is taken to be the discovery of second-harmonic generation by Fraken et al. in 1961 [1], shortly after the demonstration of the first working laser by Maiman in 1960 [2].

The nonlinear effect comes essentially from 'quantum confinement' effect. In an unconfined (bulk) semiconductor, an electron-hole pair is typically bound within a characteristic length, called the 'exciton Bohr radius'. This is estimated by replacing the positively charged atomic core with the hole in the Bohr formula. If the electron and hole are constrained further, then properties of the semiconductor change. Besides confinement in all three dimensions i.e. 'Quantum Dot' - other quantum confined semiconductors include (1) quantum wires, which confine electrons or holes in two spatial dimensions and allow free propagation in the third (2) quantum wells which confine electrons or holes in one dimension and allow free propagation in two dimensions.

Nonlinear optical effect in semiconductor quantum dots was observed in the minuscule crystals of semiconductor material composed of various compounds of chemicals such as cadmium, zinc, tellurium, selenium, sulphur, etc. of sizes less than 500 nanometers. These semiconductor nanoparticles or 'quantum dots' have been found to react to electricity or light by emitting their own light across the visible range of wavelengths from 470 to $730 \mathrm{~nm}$. 
The applications of these quantum dots include solid state lighting, with the potential to render incandescent bulbs obsolete in the future and the ability to paint lighting systems onto materials. They are used in the manufacture of medical sensors and solar cells. Thin film deposits of quantum dots on solar cell substrates substantially increase their voltage output by fluorescing the light before it is captured.

An immediate optical fe ature of colloidal quantum dots is their coloration. Such type of coloration due to embedded nanoscale metallic particles within glasses was noted in many stained glass windows in the Medieval era shape of the nanoparticles themselves', followed by Figure 1 . These rich colors in stained glass like the metallic sheen associated with naturally embedded nanoparticles in many ceramics, were appreciated and highly valued by artisans, patrons and laymen alike. Stained glass artisans sometimes treasured small vials of materials that we know were metallic oxides obtained from special mines and handed down within their families with careful instructions on how to work with them. When the size of the material particle is reduced to nanoscale, optical properties- particularly colors, can be dramatically affected. In such cases, the wavelength of light is very close to the size of the particles themselves, which causes the way that color is reflected, scattered or absorbed to be dependent on the size and shape of the nanoparticles themselves.

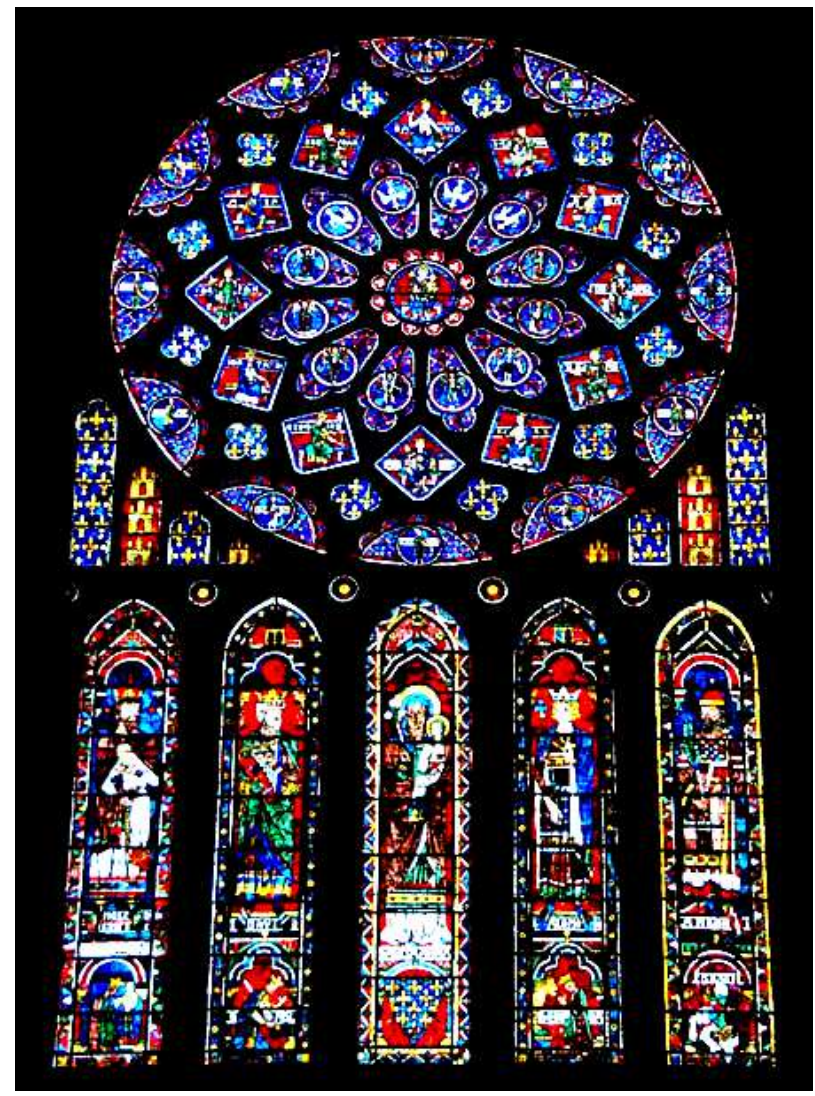

Fig. 1. Stained glass window of Chartres Cathedral 
While the material which makes up a quantum dot defines its intrinsic energy signature, the nanocrystal's quantum confined size is more significant at energies near the band gap. Thus quantum dots of the same material, but with different sizes, can emit light of different colours. The physical reason is the 'quantum confinement' effect, which will be discussed later. The larger the dot, the redder (lower energy) is its fluorescence spectrum. Conversely, smaller dots emit bluer (higher energy) light. The coloration is directly related to the energy levels of the quantum dot. Quantitatively speaking, the bandgap energy that determines the energy (and hence colour) of the fluorescent light is inversely proportional to the size of the quantum dot. Larger quantum dots have more energy levels which are also more closely spaced. This allows the quantum dot to absorb photons containing less energy, i.e., those closer to the red end of the spectrum. Recent articles in nanotechnology and in other journals have begun to suggest that the shape of the quantum dot may be a factor in the coloration as well, but as yet not enough information is available. Furthermore, it was shown that the lifetime of fluorescence is determined by the size of the quantum dot. Larger dots have more closely spaced energy levels in which the electron-hole pair can be trapped causing larger dots to show a longer lifetime. As with any crystalline semiconductor, a quantum dot's electronic wave functions extend over the crystal lattice. Similar to a molecule, a quantum dot has both a quantized energy spectrum and a quantized density of electronic states near the edge of the band gap.

A currently active area of research in non-linear optics is concerned with all-optical devices that are designed to switch and process light signals without converting them into electronic form, thus eliminating the electronic "bottleneck" in the speed of electronics used for switching, routing and signal processing. These devices are essentially based on the nonlinear optical properties of materials, i.e. changes of the refractive index caused by an intense optical beam.

When a neutral atom is placed in an electric field $\mathbf{E}$, the positively charged core and the negatively charged electron cloud surrounding it are both influenced by the electric field. The nucleus is pushed in the direction of the field and the electrons in the opposite direction. In principle, if the field is large enough it can pull the atom apart completely, 'ionizing' it. With less extreme fields, however, equilibrium is soon established, for if the center of the electron cloud does not coincide with the nucleus, leaving the atom 'polarized' with positive charge shifted slightly one way, and negative the other. The atom now has a tiny dipole moment which points in the same direction as $\mathbf{E}$ Typically, the polarization $\mathbf{P}$ (dipole moment per unit volume) is directly proportional to the field, provided $\mathrm{E}$ is not too strong. That is

$$
P=\chi E
$$

where $\chi$ is the dielectric susceptibility of the medium. The value of $\chi$ depends on the microscopic structure of the substance and also on the external electric field. Materials obeying the above equation are known as linear dielectrics. Linearity means that the displacement of the electrons is proportional to the acting force. Only under this condition the electrons oscillate sinusoidally, with amplitude proportional to the acting force (apart from a phase shift).

The above stated model of the elastically bound electron is too simple and is only an approximation, valid for small amplitudes. The deviations become significant at high oscillation energy. If this energy exceeds the value of the binding energy, the electron is set free. At this level the electron oscillates non-harmonically. Higher frequencies appear in the displacement and in the polarization $\mathbf{P}$. In this case, the linear equation between polarization and the field amplitude is no longer valid. The polarization is a complicated function of the field amplitude. It contains not only the linear term of $\mathbf{E}$ but also higher order terms. This is 
traditionally introduced by noting that the dielectric susceptibility can be expanded in a Taylor series in terms of the oscillating optical field. In an isotropic medium the general relation between the polarization $\mathbf{P}$ (dipole moment per unit volume), induced by the applied electric field $\mathbf{E}$, is expressible as a Taylor expansion involving only the magnitude, because the direction of the polarization coincides with that of the field, namely

$$
P=\left(\chi^{(1)} E+\chi^{(2)} E^{2}+\chi^{(3)} E^{3}+\ldots \ldots \ldots \ldots \ldots . . .\right)
$$

In this expansion $\chi^{(1)}$ is the normal or linear dielectric susceptibility of the medium and is related to the refractive index $\eta$ by $\eta^{2}=1+\chi^{(1)}$. It contributes to all the phenomena associated with linear optics, such as reflection, refraction, interference, etc. It is generally much larger than the nonlinear coefficients $\chi^{(2)}, \chi^{(3)}$ and so forth. In general, $\chi^{(\mathrm{n})}$, the complex dielectric susceptibilities, are tensors of rank $(n+1)$ and are related to the microscopic (electronic and nuclear) structure of the material. The even-order nonlinear optical interactions can occur only in non-centrosymmetric crystals, that is, in crystals that do not display inversion symmetry [3]. Since liquids, gases, amorphous solids (such as glass) have inversion symmetry, $\chi^{(2)}$ vanishes identically for such media and consequently they cannot produce second-order nonlinear optical interactions. On the other hand, odd-order nonlinear optical interactions can occur both for centrosymmetric and non-centrosymmetric media. Glasses possess macroscopic inversion symmetry, thereby allowing only odd-order optical nonlinearities. The third-order nonlinear optical phenomena prevail inherently in glasses because of the deficiency of long-range periodicity unlike crystals. Nonlinearity in glasses is in general quite small, but may be enhanced by several orders of magnitude by introducing metal nanoclusters in glass matrices.

The nonlinear response of the nanocluster-glass composites arises from saturable absorption at the excitonic levels. This effect drastically modifies the quantum states of the electrons and their interaction with applied optical fields; referred to as quantum confinement. Secondly, when the size of the nanoclusters is much smaller than the wavelength $(\lambda)$ of the applied optical field (i.e. for clusters with diameters less than $\lambda / 20$ ), the electric field that acts on and polarizes the free charges of these clusters can be vastly different from the macroscopic field outside the metal clusters in the surrounding medium. This polarization, in turn, modifies the dielectric constant of the composite medium. This effect is called dielectric or classical confinement. Both quantum and dielectric confinement effects alter the susceptibilities of the composite, thereby modifying the linear and nonlinear refractive indices and the absorption coefficients.

Owing to the difference in dielectric constants between the nanoclusters and the surrounding host material, local field effects arising from dielectric confinement strongly influence the optical properties and can produce major changes in optical response. Therefore, it is important to design a material which has large $\chi^{(3)}$ at the wavelength of the limited light source available and metal - doped glasses have significant possibility in this direction. The large optical nonlinearity of metal-doped glasses is considered mainly due to the local field enhancement effects on the metal microcrystallites under 'surface plasmon resonance (SPR)' condition, which mainly depends on the dielectric functions of metals and the glasses.

\section{Nonlinear optical phenomena: physical origin}

The equation for the polarization $\mathbf{P}$ links the material properties with Maxwell's equations for the propagation of electromagnetic waves. It does not give any indication as to the 
physical mechanisms which give rise to the optical nonlinearities. The origin of the nonlinear susceptibility can be viewed classically as the response of an electron driven by an electromagnetic field in an anharmonic potential well, resulting from the interatomic electric field $\mathbf{E}$ in the crystalline solid. When a light wave propagates through an optical medium, the oscillating electromagnetic field exerts a polarizing force on all the electrons comprising the medium. Because the inner electrons of the atoms are tightly bound to the nuclei, the major polarizing effect is exerted on the outer or valence electrons. The interatomic field is of the order of $10^{10} \mathrm{~V} / \mathrm{m}$. For driving optical fields $\mathbf{E}$ much weaker than that, the polarization response is essentially linear. For an intense optical field (comparable to or greater than the inter-atomic field) enough to drive the electron beyond the quadratic minimum of the interatomic potential, the response becomes increasingly nonlinear. Based on the classical atomic model of non-linearity (considering the motion of a one-dimensional anharmonic electron oscillator with damping, driven by a laser field), the ratio between the magnitudes of non-linear polarizations of successive orders can be expressed as

$$
\frac{P^{(n+1)}}{P^{n}} \approx \frac{e|E|}{m \omega_{0}^{2} r} \approx \frac{e|E|}{e\left|E_{a t}\right|}
$$

where $\omega_{0}$ is the oscillation frequency of the electron and $r$ is the radius of the equilibrium orbital of the electron $\left(m \omega_{0}^{2} \mathrm{r}\right.$ is actually the expression for the linear force which is equal to $\mathrm{e}\left|\mathrm{E}_{\mathrm{at}}\right|$, where $\mathrm{E}_{\mathrm{at}}$ is the intra-atomic electric field at a distance equal to Bohr radius $\mathrm{a}_{0}$ ). Now, $E_{a t}=\frac{e}{a_{0}^{2}}=5.15 \times 10^{11} \mathrm{~V} / \mathrm{m}$. Following the expression for energy flux density of an electromagnetic wave $\left(=\varepsilon c E^{2}\right)$, the above value of $E_{a t}$ corresponds to a laser power of $7 \times 10^{20}$ Watt $/ \mathrm{m}^{2}$, where $\varepsilon$ (dielectric constant of vacuum) $=8.8 \times 10^{-12}$ and $c$ (speed of light $)=3 \times 10^{8}$, expressed in SI units.

At low light intensities, the optical fields are a small fraction of the molecules' total internal fields $\left(\sim 5 \times 10^{11} \mathrm{Vm}^{-1}\right)$. At high photon intensities, the induced fields become a significant fraction of the total. This added internal field substantially modifies the motions of the charges within the molecule and alters the nature of the polarization within the materials. Also, this added internal field "anharmonically" modifies the light wave's propagation, leading to remarkable effects when they occur coherently, constructively. That is when a material's properties (e.g. the interaction length for a process and the strength of the perturbation) are well-matched (i.e. phase-matched) to the laser wavelength, second- and third-order non-linear optical effects can drastically change the colour content or manner of propagation of light. Therefore, the non-linear phenomenon is evidenced by the changes in optical properties as the intensity of light is increased.

We thus expect that under conditions of non-resonant excitation the second-order susceptibility $\chi^{(2)}$ will be of the order of $\chi^{(1)} / E_{a t}$ [4]. For condensed matter $\chi^{(1)}$ is of the order of unity, and hence we expect that $\chi^{(2)}$ will be of the order of $1 / E_{a t}$ or

$$
\chi^{(2)} \cong 5 \times 10^{-8} \mathrm{~cm} / \text { statvolt }=5 \times 10^{-8}\left(\mathrm{~cm}^{3} / \mathrm{erg}\right)^{1 / 2}=5 \times 10^{-8} \text { esu }
$$

Similarly, we expect $\chi^{(3)}$ to be of the order of $\chi^{(1)} / \mathrm{E}_{\mathrm{at}}{ }^{2}$, which for condensed matter is of the order of 


$$
\chi^{(3)} \cong 3 \times 10^{-15} \mathrm{~cm}^{2} / \text { statvolt }^{2}=3 \times 10^{-15} \mathrm{~cm}^{3} / \mathrm{erg}=3 \times 10^{-15} \mathrm{esu}
$$

These predictions are in fact quite accurate, as one can see by comparing these values with the actual measured values of $\chi^{(2)}$ and $\chi^{(3)}$. For certain purposes, it is useful to express the second- and third-order susceptibilities in terms of fundamental physical constants. Noting that the number density $\mathrm{N}$ of the condensed matter is of the order $\left(a_{0}\right)^{-3}$, we find that

$$
\chi^{(2)} \cong h^{4} / 16 \pi^{4} m^{2} e^{5} \text { and } \chi^{(3)} \cong h^{8} / 2^{8} \pi^{8} m^{4} e^{10}
$$

The most common procedure for describing nonlinear optical phenomena is based on expressing the polarization $\mathbf{P}(\mathrm{t})$ in terms of the applied electric field strength $\mathbf{E}(\mathrm{t})$ as we have done in Eq (2). The reason why the polarization plays the key role in the description of nonlinear optical phenomena is that a time-varying polarization can act as the source of new components of the electromagnetic field. The wave equation in nonlinear optical media often has the form [4]

$$
\nabla^{2} E-\frac{\eta^{2}}{c^{2}} \frac{\partial^{2} E}{\partial t^{2}}=\frac{4 \pi}{c^{2}} \frac{\partial^{2} P^{N L}}{\partial t^{2}}
$$

where $\eta$ is the usual linear refractive index and $c$ is the speed of light in vacuum. We can interpret this expression as an inhomogeneous wave equation in which the polarization $\mathbf{P}^{\mathrm{NL}}$ associated with the nonlinear response drives the electric field $\mathbf{E}$ This equation expresses the fact that, whenever $\partial^{2} P^{N L} / \partial t^{2}$ is non-zero, charges are being accelerated, and according to Larmor's theorem from electromagnetism, accelerated charges generate electromagnetic radiation. It should be noted that the power series expansion, expressed by Eq. (2), need not necessarily converge. In such circumstances the relationship between the material response and the applied electric field amplitude must be expressed using different procedures.

The physical origin of nonlinear optical phenomena can be categorized as either structural or compositional [5]. Here 'structural' refers to light induced structure changes, such as a change of electronic density, average interatomic distances, molecular orientation, phase transition, etc. These phenomena belong to the intrinsic category. The 'compositional' refers to light-induced chemical composition changes such as molecular dissociation, polymerization etc. Intrinsic nonlinearity violates the principle of superposition arising from a nonlinear response of the individual molecule unit cell to the fields of two or more light waves, while extrinsic nonlinearity is related to changes in the composition of the medium that results from the absorption or emission of light. In either type of nonlinearity, the optical properties of the medium depend on the intensity of light and the order of nonlinearity can be classified according to the power of the intensity involved.

Colloidal suspension of metal nanoclusters in centro-symmetric dielectric materials (such as glasses) has recently attracted immense interest in materials science for their potential photonic applications. Various types of glasses are very attractive materials for these applications, because of their relatively fast response time. Furthermore, glass-based nanocomposites are, in general, expected to play an important role as materials for various nanotechnology applications, due to low cost, ease of processing, high durability, resistance and high 
transparency, as well as the possibility of tailoring the behavior of glass-based structures. Since the first attempt of Faraday to explain the nature of the colour induced in glasses by small metallic precipitates, many studies have been dedicated to the properties of metal nanocluster composite glass. In general, the physical properties of these systems change dramatically in the transition from atom to molecule to cluster to solids, where the cluster regime is characterized by the confinement effects, causing thereby these systems particularly interesting. In the last decade, metal nanoclusters embedded in insulating matrices, have received increasing interest due to their peculiar optical, magnetic and catalytic properties when the size becomes comparable to or less than the electronic mean free path. The nonlinear susceptibility $\chi^{(n)}$ of any material is intimately related to its electronic structure. Therefore, it is of utmost importance to understand the relationship between the nonlinearity and electronic structure and thereby the chemical structure of the material so that a predictive capability can be developed for designing device grade materials.

\section{Optical properties of metal nanoparticles}

Metals contain free electrons which are responsible for their electrical conduction. If a spatial variation of the density of freely moving electrons with respect to the background of positive ions is created, the restoring forces are formed that tend to pull the electrons back to their initial position. Since the electrons have inertia, their distribution overshoots the equilibrium position creating a new charge distribution and with it the restoring forces. The electron charge density thus continues an oscillating motion. Such a system where oppositely charged particles can move freely with respect to each other is called plasma and their oscillatory motion the plasma oscillation. Quantization of the plasma oscillations leads to quasi-particles or the plasmons. Localized surface plasmons or 'surface polaritions' are collective electron charge oscillations in metallic nanoparticles that are excited by light. They exhibit enhanced near-field amplitude at the resonance wavelength. This field is highly localized at the nanoparticle and decays rapidly away from the nanoparticle/dieletric interface into the dielectric background, though far-field scattering by the particle is also enhanced by the resonance. Light intensity enhancement is a very important aspect of localized SPRs and the localization means that these SPRs have very high spatial resolution, limited only by the size of nanoparticles. Figure 2 shows the Schematic of plasma oscillation for a metallic sphere, indicating displacement of the conduction electron charge cloud relative to the nuclei.

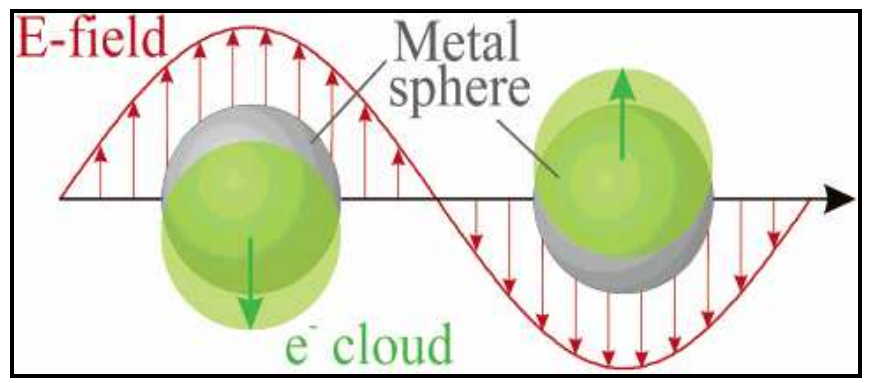

Fig. 2. Schematic of plasma oscillation for a sphere, showing displacement of the conduction electron charge cloud relative to the nuclei. 
Assuming the electron charge density $\rho$ to be a continuous function of position and using classical electrodynamics, an equation of motion for $\rho(x, t)$ can be written as

$$
\frac{\partial^{2} \rho(x, t)}{\partial t^{2}}=-\frac{4 \pi}{m} \frac{\Delta N}{\Delta V} e^{2} \rho(x, t)
$$

where $\Delta N$ is the number of electrons in volume element $\Delta V$ while other quantities have their usual meanings. Eq. (4) represents simple harmonic motion with a bulk plasma oscillation frequency

$$
\omega_{p}=\sqrt{\frac{4 \pi n e^{2}}{m}}
$$

where $n=\Delta N / \Delta V$ is the average electron density.

For a metal (complex dielectric function $\varepsilon(\omega)$ ) nanoparticles of radius $R$ embedded in a host of dielectric constant $\varepsilon_{d}$, the plasmon response in quasi-static approximation $(R<\lambda$, wavelength of light) is essentially dipolar in nature. The dipole plasmon response of the metal nanoparticles is defined by its polarizability

$$
\alpha=4 \pi \varepsilon_{0} R^{3}\left|\frac{\varepsilon(\omega)-\varepsilon_{d}}{\varepsilon(\omega)+2 \varepsilon_{d}}\right|
$$

The resonance will be observed when the real part $\varepsilon^{\prime}(\omega)$ of the complex dielectric function $\varepsilon(\omega)$ satisfies the condition

$$
\varepsilon^{\prime}(\omega)=-2 \varepsilon_{d}
$$

and its imaginary part $\varepsilon^{\prime \prime}(\omega)<<1 . \varepsilon^{\prime}(\omega)$ is negative when the frequency of electromagnetic wave $\omega$ is below the bulk plasmon frequency $\omega_{p}$. In Drude model, $\varepsilon^{\prime}(\omega)$ and $\varepsilon^{\prime \prime}(\omega)$ can be approximated in terms of $\omega_{p}$ and plasma relaxation frequency $\Gamma$ as

$$
\begin{gathered}
\varepsilon^{\prime}(\omega)=1-\frac{\omega_{p}^{2}}{\omega^{2}+\Gamma^{2}} \\
\varepsilon^{\prime \prime}(\omega)=1-\frac{\omega_{p}^{2} \Gamma}{\omega\left(\omega^{2}+\Gamma^{2}\right)}
\end{gathered}
$$

Eqs. (7) and (8) yield the Drude resonance frequency as

$$
\omega_{D}=\frac{\omega_{P}}{\sqrt{2 \varepsilon_{d}+1}}
$$

The plasma frequency $\omega_{p}$ is scale-invariant but the Drude free electron response varies with geometric shape of the particle. Drude free electron model does not yield the surface plasmon frequency for metal nanoparticles of interest correctly. This is mainly because of the additional electric susceptibility introduced by interband transitions between the 
outermost $d$ - and $s$ - orbitals at optical frequencies. These electronic excitations couple strongly with the free electron response yielding large changes in $\varepsilon^{\prime}(\omega)$ and $\varepsilon^{\prime \prime}(\omega)$. In addition, these can produce significant damping mechanism for plasmon decay which will reduce the quality of the optical resonance. In case of silver nanoparticles, these interband transitions are responsible for the frequency difference between the theoretically predicted Drude response at $\omega_{D}=5.6 \mathrm{eV}$ and the observed surface plasmon resonance at $\omega_{s p}=3.6$ $\mathrm{eV}$. More accurate model for the optical properties of metal nanoparticles can be obtained using Mie theory based on Maxwell's equations. Results of Mie theory clearly predict the experimental optical spectra of metal nanoparticles. This theory has also been used to estimate the variations in the electrodynamic response due to the size and anisotropy. It can also account for the optical response of metal nanoparticles beyond the quasi-static limit $(2 R / \lambda>0.05)$, whose plasmon resonances are red-shifted by phase retardation and broadened by radiation damping. Higher-order plasmon modes can also become significant. For example, quadrupole resonance in silver and gold nanoparticles can be observed in optical extinction spectra at sizes $\geq 60 \mathrm{~nm}$. For spherical metal clusters, Mie plasmons considering $\varepsilon_{d}=1$ are shown to occur at frequency [6]

$$
\omega_{l}=\omega_{p} \sqrt{\frac{l}{2 l+1}}
$$

where $l$ is the angular momentum of plasmon mode. Using a single photon of appropriate energy one can excite only $l=1$ dipole plasmon oscillations in a metallic cluster.

The dipole surface plasmons are responsible for the formation of giant resonances in photoabsorption spectra of metal clusters [7-10]. They also play an important role in inelastic collisions of charged particles with metal clusters [11-13]. It was also demonstrated that collective excitations make a significant contribution to the electron energy loss spectrum in the region of the surface plasmon resonance. One-photon absorption cross-section in the plasmon approximation is shown to be [14]

$$
\sigma_{1}=\frac{4 \pi N e^{2}}{m c} \frac{\omega^{2} \Gamma_{1}}{\left(\omega_{1}^{2}-\omega^{2}\right)^{2}+\omega^{2} \Gamma_{1}^{2}}
$$

where $\Gamma_{1}$ is Landau damping. Connerade et al. [13] have also shown that the two-photon absorption can occur for $l=2$ i.e. quadratic mode and is not allowed for $l=1$ plasmon mode (dipolar plasmon). Two-photon absorption cross-section is given by [13]

$$
\sigma_{2}=\left(\frac{4 \pi N e^{2}}{m c}\right)^{2} \frac{A^{2} \hbar}{2 \pi \omega_{1} N \Delta R^{2}} \frac{\omega^{2}}{\omega_{1}^{2}} \frac{1}{\left(\left(\omega-\omega_{1}\right)^{2}+\frac{\Gamma_{1}^{2}}{4}\right)} \frac{\Gamma_{2}}{\left(\left(\omega_{2}-2 \omega\right)^{2}+\frac{\Gamma_{2}^{2}}{4}\right)}
$$

where $A \approx 2.79$ and $\Delta R$ is the width of the domain in the vicinity of the cluster surface within which plasmon excitations take place. Note that unlike $\sigma_{1}, \sigma_{2}$ depends on $\hbar$. It is because $l=1$ plasmon oscillations are purely classical effect, while $\sigma_{2}$ arises from the interaction between dipole and quadrupole plasmon modes and the two-photon absorption profile differs substantially from the one-photon case. Connerade et al. [13] have also shown that, in addition to dipole plasmon excitations multipole plasmons 
(quadrapole, octupole, etc) contribute to the multiphoton excitation process, which results in a significant difference of plasmon resonance profiles between the cross-sections for multiphoton and single-photon absorption. The results were obtained within a theoretical model for the multiphoton excitation of a jellium cluster. This model is applicable to metal clusters.

In general, when an electromagnetic wave propagates in the composite medium, it does not resolve the individual scattering centers, and the medium appears as a homogeneous medium characterized by an effective complex dielectric function. If we consider a composite consisting of small particles occupying a relative volume fraction $p<<1$ in a dielectric host, the complex dielectric constant of the metal cluster, which is a function of the frequency of the incident electromagnetic field by $\varepsilon_{m}(\omega)=\varepsilon_{1}(\omega)+i \varepsilon_{2}(\omega)$. The optical response of the metal nanocluster glass composite systems may be approached following two classes of models, namely, (i) discrete island model, in which each scattering center is considered, and (ii) effective medium theory, in which one scattering center is considered, the rest of the surrounding medium being averaged into a homogeneous medium. Considering the first kind of approach, Maxwell-Garnet [15] considered that the metal clusters embedded in a host matrix of dielectric constant $\varepsilon_{\mathrm{h}}$ are polarized by an optical field. The Lorentz local-field relationships show that the effective dielectric constant $\varepsilon_{\text {eff }}$ of such a composite medium is given by

$$
\frac{\varepsilon_{\text {eff }}-\varepsilon_{h}}{\varepsilon_{\text {eff }}+2 \varepsilon_{h}}=p \frac{\varepsilon_{m}-\varepsilon_{h}}{\varepsilon_{m}+2 \varepsilon_{h}}
$$

For small volume fractions, Eq. (13) may be expanded to the first order in the volume fraction. The optical absorption coefficient is related to the imaginary part of $\varepsilon_{\text {eff. }}$ Mie was the first to derive an exact description of the optical absorption and elastic scattering by a collection of metal spherical clusters suspended in a transparent medium. By adding the contribution of single clusters, the theory accounts correctly for many experimental cases, provided that interaction effects among the clusters as well as multiple scattering effects are missing. This is concerned, however, with a large class of inhomogeneous composite glasses, where the cluster density is anyhow sufficiently low to allow a description within this regime. The optical absorption coefficient $\alpha$ of a collection of uniform spheres, very small compared to the wavelength $\lambda$ of light and embedded in a medium of refractive index $\eta_{d}$, is well described by the Mie scattering theory. The general expression for the extinction coefficient, which includes both absorption and scattering, is given by [16]

$$
\alpha=p \frac{18 \pi n_{d}{ }^{3}}{\lambda} \frac{\varepsilon_{2}}{\left(\varepsilon_{1}+2 n_{d}{ }^{2}\right)^{2}+\varepsilon_{2}^{2}}
$$

The extinction coefficient in expression (14) has a maximum at $\lambda$ for which the condition $\left(\varepsilon_{1}+2 n_{d}{ }^{2}\right)=0$ is satisfied. This is due to the absorption resonance known as the surface plasmon resonance (SPR). The UV-Visible spectrum of a $\mathrm{Cu}^{+}$- implanted glass sample shows such a typical SPR band at $589 \mathrm{~nm}$ (Fig. 3). 


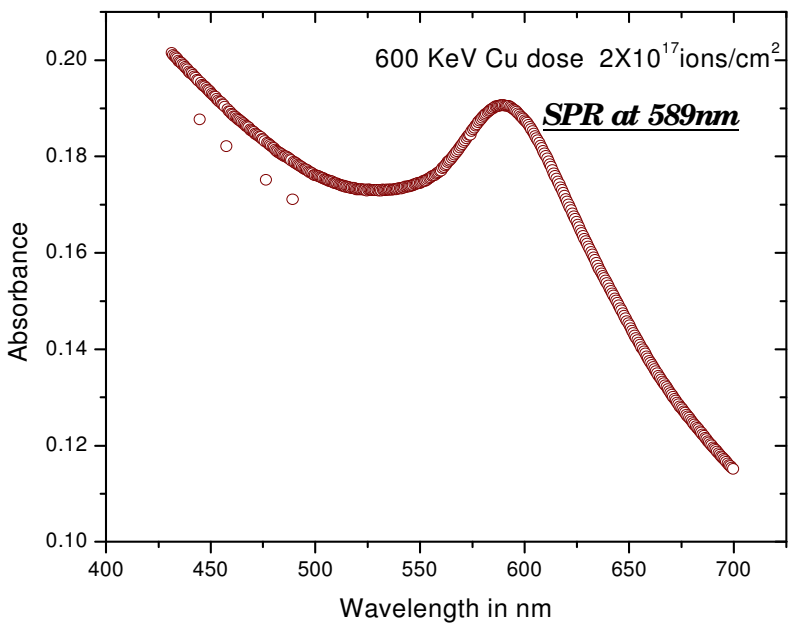

Fig. 3. Optical absorption band for copper nanoclusters in glass

Plasmons are the quanta associated with longitudinal waves propagating in matter through the collective motion of conduction band electrons. Maxwell's equations tell us that an interface between a dielectric (e.g. silica glass) and a metal (e.g. Ag or Au) can support a surface plasmon (SP). An SP is a coherent electron oscillation that propagates along the interface together with an electromagnetic wave. These unique interface waves result from the special dispersion characteristics (dependence of dielectric constant on frequency) of metals. What distinguishes SPs from 'regular' photons is that they have a much smaller wavelength at the same frequency. For example, a HeNe laser, whose free-space emission wavelength is $633 \mathrm{~nm}$, can excite an SP at a Si/Ag interface with a wavelength of only 70 $\mathrm{nm}$. When the laser frequency is tuned very close to the SP resonance, SP wavelengths in the nanometer range can be achieved. The short-wavelength SPs enable the fabrication of nanoscale optical integrated circuits, in which light can be guided, split, filtered, and even amplified using plasmonic integrated circuits that are smaller than the optical wavelength. The position, width and shape of the SPR band are determined by the metal dielectric function, also on the size, shape and concentration of the particles and on the surrounding dielectric medium. The position of the resonant absorption band changes with the cluster size, but remains more or less unchanged for the clusters with less than $10 \mathrm{~nm}$ diameter, as was predicted by Mie in his scattering theory. Moreover, quantum corrections to the classical absorption become significant as the cluster dimension approaches $1 \mathrm{~nm}$ diameter [17]. Equation (14) gives a band of Lorentzian shape: if the band is narrow, its width $w$ at half maximum absorption (FWHM), i.e. bandwidth, is $\lambda_{m}^{2} / \lambda_{a}$ and is given by

$$
\begin{gathered}
w=\frac{\lambda_{m}^{2}}{\lambda_{a}} \\
=\left(\varepsilon_{0}+2 n_{d}^{2}\right) c / 2 \sigma
\end{gathered}
$$


The d.c. conductivity, $\sigma$, is given by

$$
\sigma=\frac{N_{e} e^{2} R}{m u_{F}}
$$

where $R$ is the particle radius, $u_{F}=\left(\frac{2 E_{F}}{m}\right)^{1 / 2}$ is the electron velocity at the Fermi energy $E_{F}=\frac{h^{2}}{2 m}(3 n 8 \pi)^{2 / 3}$ and $N_{\mathrm{e}}$ is the number of electrons per unit volume. Therefore, the experimental result of constant band position is reasonable, because $\lambda_{m}$ does not depend upon the conductivity, $\sigma$, which is determined by the mean free path of the electrons and therefore, upon the particle size. Alternatively, Equation (15) shows that the dependence of bandwidth upon particle size, i.e. $w \propto 1 / R$, as obtained from Equations (15) and (16), results from the changing mean free path of the electrons. Figure (4) gives a comparison between the experimentally measured absorption band for silver particles of about $10 \mathrm{~nm}$ diameter, suspended in glass, and the calculated one from Equation (14), using the measured $\lambda_{m}$ and bandwidth [18]. The agreement between these curves confirms that the absorption results from the free electrons in the silver particles. The slight increase in absorption at lower wavelengths probably results from the ultraviolet irradiation of the cerium present in the glass matrix [19].

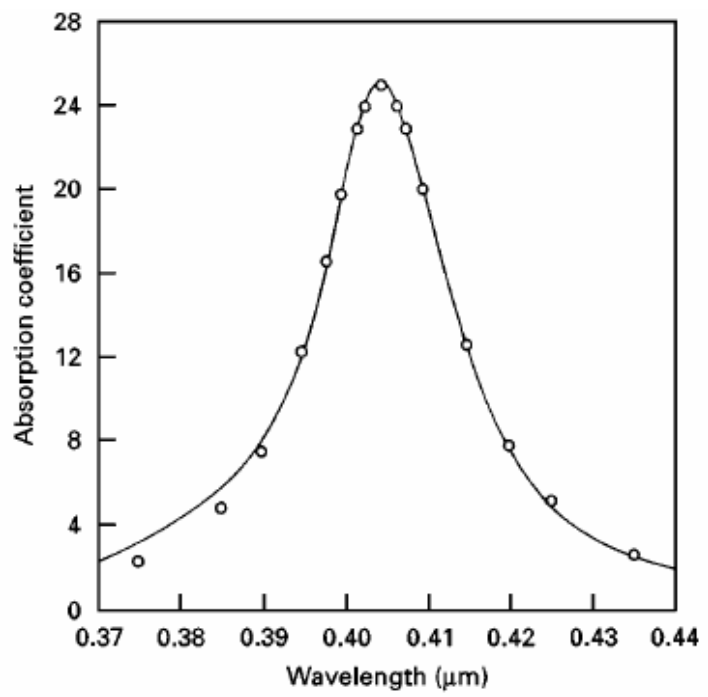

Fig. 4. (o) Measured and (__ calculated absorption bands for silver nanoparticles of about $10 \mathrm{~nm}$ diameter, suspended in glass

Eq. (14) predicts a shift of the resonance to longer wavelengths and increasing half-widths for the particles of radius $R \geq 10 \mathrm{~nm}$. Figure (5) shows the optical extinction per unit $\mathrm{Ag}$ concentration as a function of wavelength for various values of colloidal radius. 


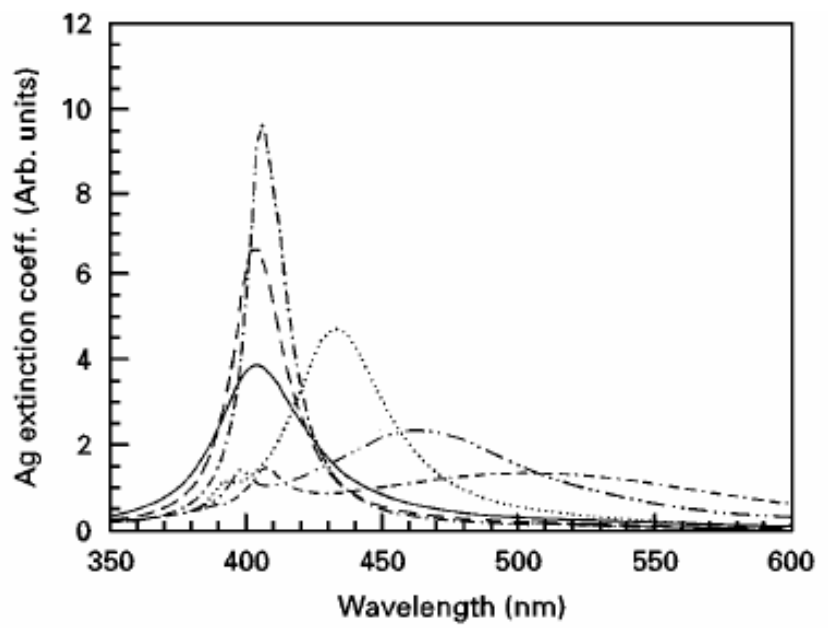

Fig. 5. Mie extinction coefficient of silver colloids of various radii as a function of wavelength of the incident light. R (nm): ( _ ) 2.5, (_ _ ) 5, (_ . ) 10, (.....) 25, (_ .. _ ) 35, $\left({ }_{-} \cdot-\_\right) 45$.

The average radius of metal spheres, small compared to the wavelength of light, can be approximately estimated from the resonance optical absorption spectrum as per the Mie scattering formula.

$$
r_{\text {metal }}=\frac{v_{F}}{\Delta \omega_{1 / 2}}=\frac{v_{F}}{2 \pi c_{0}\left(\frac{\Delta \lambda}{\lambda_{p}^{2}}\right)}
$$

Where $v_{F}=$ Fermi velocity and $\Delta \omega_{1 / 2}$ is the full width at half maximum (FWHM) of the SPR absorption when plotted as a function of angular frequency $\omega, c_{0}$ is the speed of light in vacuum, $\lambda_{p}$ is the wavelength where absorption peak appears, and $\Delta \lambda$ gives the bandwidth. This expression [20], however, provides a very crude estimate of the metal particle dimension as it is reliable only when the metal colloids are strictly spherical. It is also difficult to estimate the particle dimensions from the optical absorption spectra as the main features of the SPR bands (i.e wavelength, position and FWHM) depend on size, size distribution, shape, lattice parameters and filling factors of the metal particles.

As the dielectric constant is intensity dependent, the corresponding refractive index is also intensity dependent. The intensity dependent refractive index $\eta$ is usually expressed as

$$
\eta=\eta_{0}+\eta_{2} I
$$

where $\eta_{0}$ is the linear refractive index of the material and is a function of $\chi^{(1)}$ [21]. I is the intensity of light averaged over a period and $\eta_{2}$ is the nonlinear refractive index of the material. The total absorption, $\alpha$, under saturation can be expressed as [22]:

$$
\alpha=\alpha_{0}+\beta I
$$


$\beta$ is the coefficient characterizing the nonlinear absorption and is known as the two-photon absorption coefficient'. For all-optical devices, the nonlinear refractive index and the twophoton absorption play critical roles. The nonlinear refractive index and the two-photon absorption coefficient are related to the real and imaginary parts of $\chi^{(3)}$ according to the following equations:

$$
\begin{aligned}
& \eta_{2}=12 \frac{\pi \operatorname{Re}\left[\chi^{(3)}\right]}{\eta_{0}} \\
& \beta=96 \frac{\pi^{2} \omega \operatorname{Im}\left[\chi^{(3)}\right]}{\eta_{0}^{2} c^{2}}
\end{aligned}
$$

Eqs. (20) and (21) are valid in the case of negligible linear absoption, i.e. when $\alpha_{0} / 2 \mathrm{kn}_{0}<<1$, where $\mathrm{k}=2 \pi / \lambda$.

\section{Ion beam synthesis of metal - glass nanocomposites}

Among different possible synthesis processing, ion-beam based techniques proved to be very suitable in synthesizing metal nanocluster glass composites. Moreover, the composition of the clusters can be varied easily by sequential ion implantation in the matrix of two different elements whose energy and dose can be tailored so as to maximize the overlap between the implanted species and to control their local relative concentration. Ion implantation has been shown to produce high-density metal colloids in glasses and other materials. The high-precipitate volume fraction and small size of nanoclusters in glasses lead to the generation of third-order susceptibility much greater than those for metal-doped solids. This has stimulated interest in the use of ion implantation to make nonlinear optical materials. An attractive feature of ion implantation to form these nanoclusters, compared to the classical technique of melt-glass fabrication process, i.e. mixing selected metal powder with molten glass, is that the linear and nonlinear properties occur in a well-defined space in an optical device, and by using focused ion beams, point quantum confinement may be accomplished. Although several other methods, such as ion-exchange [23], sol-gel [24], electrolytic colouration, etc., also exist for introducing the metal into the insulating substrates, the ion implantation has the following advantages of being a generally applicable process; (a) it can be performed at an ambient temperature, (b) it has no side diffusion problem, (c) it offers an accurate control of the total number of ions being added to the target and (4) it offers a predictable depth distribution in the target matrix determined by the incident ion beam energy.

Ion implantation in glassy structures yields in the precipitation of metal colloids at a reasonably higher local concentration because of the large specific volume and more open structure of the glassy state relative to that of the crystalline counterpart. Metal nanoclustres can be easily incorporated within the open spaces present in the glass matrix. Figure (6) shows the schematic structure of $\mathrm{SiO}_{2}$ in crystalline and glassy form.

Ion implantation of metals into glass has been explored since the last decade as a useful technique to produce nanocomposite materials in which nanometer-sized metal or semiconductor particles are embedded in dielectric matrices. Modifications induced by ion implantation depend on the glass composition as well as on the ion species, and the fluence, energy and the temperature of the process. Both nuclear and electronic processes give rise to 


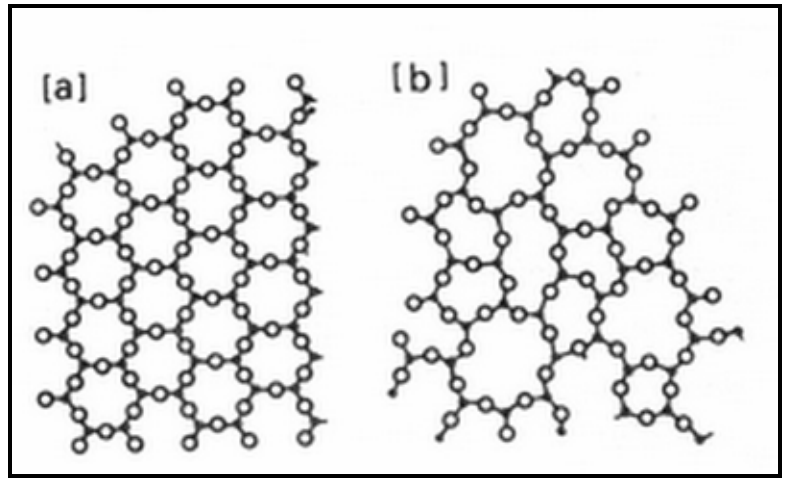

Fig. 6. Schematic 2-D representation of the structure of $\mathrm{SiO}_{2}(\mathrm{a})$ crystalline (b) glassy form

the structural changes in materials, and several evidences of clusterisation of metal atoms implanted in glass matrices are reported in literatures [25-32]. Alternatively, low-mass ionbeams can be used for promoting cluster aggregation in surface layers of glass previously doped with metal ions by other suitable techniques. High energy ion beam mixing [33] and ion beam assisted deposition [34] have also been successfully exploited for the preparation of clusters-doped silica glass. Figure (7) sketches some of these methods [29]. Furthermore, ion implantation has been used as the first step of combined methodologies that involve other treatments such as thermal annealing in controlled atmosphere, laser or ion irradiation [35-39]. Heat treating the implanted sample reduces the strains and charge imbalances caused by implantation. Moreover, heating increases the diffusion coefficient and the implanted atoms move to the lower energy metallic state of the clusters increasing the localized volume fraction.

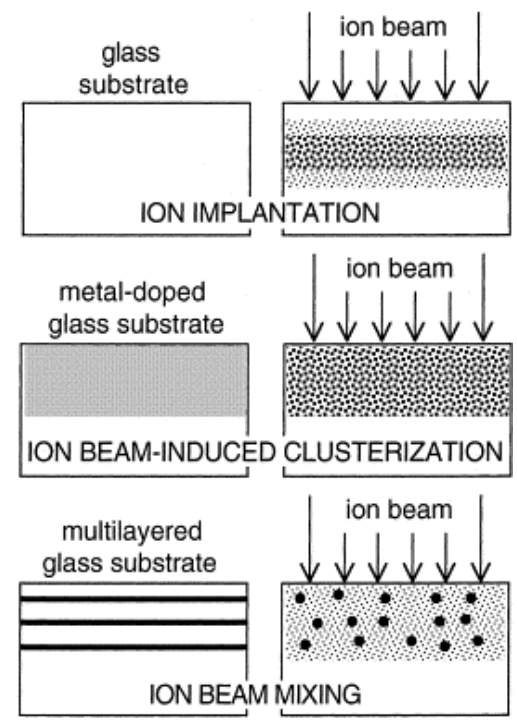

Fig. 7. Ion beam - based methods for promoting metal cluster formation in silica glass 
The physical mechanisms governing the cluster formation are presently under debate. Hosono discussed [40] the chemical reactivity of the species involved in the implants on $\mathrm{SiO}_{2}$ glasses. In the case of weak or negligible chemical interaction, the elements which do not react with the matrix (for example, $\mathrm{Ag}, \mathrm{Cu}, \mathrm{Au} . .$. ) are considered to directly form metallic precipitates under appropriate conditions. Among those which tend to form bonds with $\mathrm{O}$ from the silica network, so forming a defective Si-Si homobond, the cluster formation is predicted when the Gibbs energy for metal oxide formation is greater than that of $\mathrm{SiO}_{2}$. The comparison of the two energies should be made at the extrapolated effective temperature of $3000 \mathrm{~K}$. The validity of this criterion as a first approximation is good, yet not giving account for the effect of the concentration of the implanted species, nor providing a criterion for fixing the local temperature at which the two energies must be compared. A more general approach is due to Hosono, Matsunami and Imagawa [41, 42]. Starting from the study of the implantation-induced defects in silica, they pointed out the importance of the chemical interaction strength among atoms coming into play in the creation of defects. Although a vast volume of literature is devoted to the nonlinear optical studies in metal nanocluster glass composites, we will discuss here only those works which give meaningful insights into the study of third-order optical nonlinearity; its correlation with electronic and chemical structure and those which offer some useful guidelines for developing potential nonlinear optical materials for photonic applications.

Nanocluster formation in metal-ion implanted silica and glasses has been studied by using different techniques. Out of various metal nanoparticles, silver, copper and gold are extensively studied in semicontinuous thin films, colloids and in different glass matrices for their nonlinear optical properties [43]. Different metal alloy nanoclusters [44, 45] are also studied in this aspect. Optical absorption measurements [46-48] and transmission electron microscopy (TEM) give information on the cluster shape, size and crystalline state. Crosssectional TEM measurements give the details of the colloid size distribution as a function of depth [48, 49]. Rutherford Backscattering Spectrometry (RBS) and Secondary Ion Mass Spectrometry (SIMS) can be used to obtain the depth distribution of the implanted species. X-ray photoelectron spectroscopy (XPS) and Auger electron spectroscopy (AES) determine the chemical state of the implanted ions. Degenerate four wave mixing (DFWM) [50] and Zscan [51] methods have been used for measurements of $\chi^{(3)}$ of the metal doped glasses.

Ricard et. al [50] were the first to measure the non-linear properties of such colloids (silver, gold) in bulk silica and other workers [42-55] extended these measurements. The non-linear index $\eta_{2}$ of $160 \mathrm{keV}$ copper-implanted silica at a fluence of $10^{17}$ ions $/ \mathrm{cm}^{2}$ was determined using the Z-scan method and was found to be around $4 \times 10^{-14} \mathrm{~m}^{2} \mathrm{~W}^{-1}$ [42]. More recently, the formation of various metal colloids (copper, silver, gold, lead, tin, iron, phosphorus etc.) in silica glasses by ion implantation under various conditions and measurements of $\chi^{(3)}$ values in these cases have been reported [53-56]. The highest value of $\chi^{(3)}\left(3 \times 10^{-6}\right.$ e.s.u $)$ was obtained, so far, for tin implanted $\left(2 \times 10^{17}\right.$ ions $/ \mathrm{cm}^{2}$ dose $)$ silica glass [57] at a wavelength of $500 \mathrm{~nm}$; corresponding to surface plasmon resonance (SPR) of tin. Figures (8) and (9) show the TEM images of such microstructures. As shown in these figures, the implanted ions form spherical nanoclusters. The crystallinity of the nanoclusters is evidenced [57] from X-ray diffraction studies, as shown in Fig. 10. All the XRD peaks are identified as those of metallic tin crystallites and relative peak intensities confirm that there is no evidence of preferential orientation of the microcrystallites. 


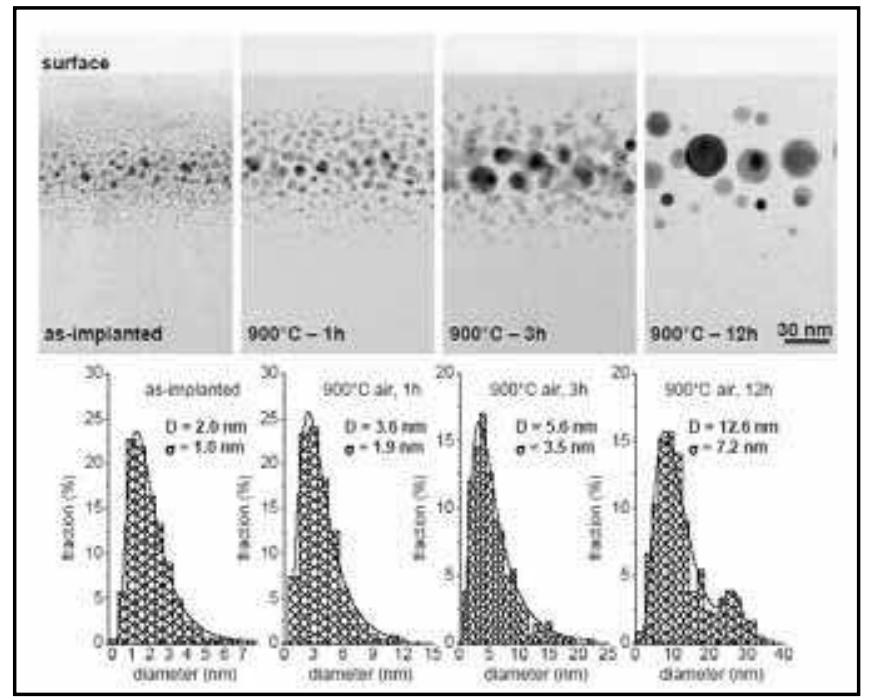

Fig. 8. TEM results on Au-implanted silica samples annealed in air at $900{ }^{\circ} \mathrm{C}$ for different time intervals: cross-sectional bright-field micrographs with the corresponding histogram of size distribution for the as-implanted and annealed for $1 \mathrm{~h}, 3 \mathrm{~h}, 12 \mathrm{~h}$ samples.

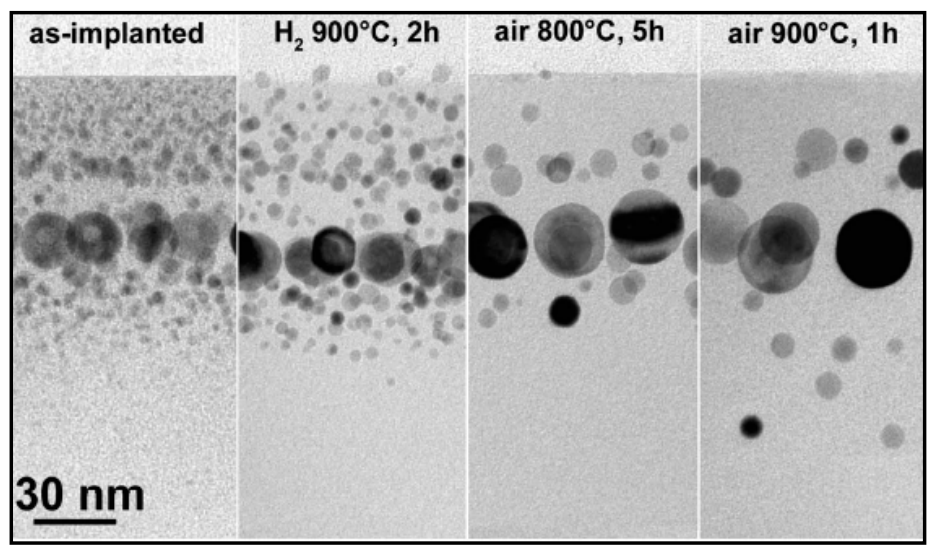

Fig. 9. Cross-sectional bright-field TEM images of sequentially ion implanted silica with Pd (130 keV) and $\mathrm{Ag}(130 \mathrm{keV})$ at dose $3 \times 10^{16}$ ions $/ \mathrm{cm}^{2}$ : (a) as implanted; (b) annealed in reducing atmosphere at $9000 \mathrm{C}$ for 2 hours; (c) annealed in air at $8000 \mathrm{C}$ for 5 hours; (d) annealed in air at $9000 \mathrm{C}$ for 1 hours. 


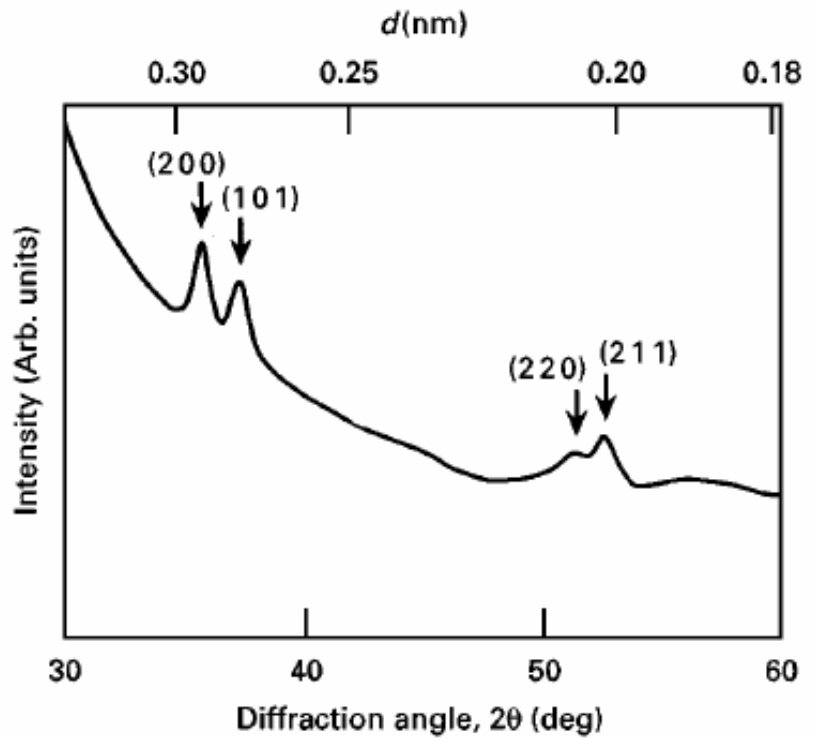

Fig. 10. XRD pattern of $\mathrm{Sn}^{+}$-implanetd silica glass

Another example of changing both the linear and nonlinear optical properties of silica by implanting 2.0 MeV copper, $350 \mathrm{keV}$ tin, $1.5 \mathrm{MeV}$ silver and $3.0 \mathrm{MeV}$ gold have been studied by D. Ila et. al [58]. Resonance enhancement of $\chi^{(3)}$ with in-diffusion of gold was exploited for high non-linearity at wavelength above $1000 \mathrm{~nm}$ [59]. Another significant work reported [60] of copper nanoparticles prepared by the reduction of copper (II) acetate in water and 2ethoxyethanol using hydrazine under reflux. The synthesized nanoparticles exhibited a distinct absorption peak in the region 572-582 nm. The nonlinear optical properties of the copper colloids were first measured using the $Z$-scan technique and $\chi^{(3)}$ values obtained were found to be of the order of 10-11-10-12 esu. Recently reported works includes synthesis of metal nanocluster composite glass by $\mathrm{Cu}$ ions implanted into silica with dose of $1 \times 10^{17}$ ions/cm [61, 62]. Third-order nonlinear optical properties of the nanoclusters were measured at $1064 \mathrm{~nm}$ and $532 \mathrm{~nm}$ excitations using Z-scan technique. Absolute third-order nonlinear susceptibility $\chi^{(3)}$ of this kind of sample was determined to be $2.1 \times 10^{-7}$ esu at $532 \mathrm{~nm}$ and $1.2 \times 10^{-7} \mathrm{esu}$ at $1064 \mathrm{~nm}$. Nonlinear optical properties of Ag-Cu nanoclusters co-doped in $\mathrm{SiO}_{2}$ substrate prepared using the sol-gel technique were investigated under the influence of the second harmonic of a high-power nanosecond Nd:YAG laser [63]. Thirdorder nonlinear susceptibility $\chi^{(3)}$ was determined to be of the order of $10^{-7}$ esu and nonlinear refractive index was found to be of the order of $10^{-9} \mathrm{~cm}^{2} /$ Watt. The measured nonlinear refractive indices $\left(\sim 0^{-6}\right.$ e.s.u.) for these microcrystallite-doped glass composites compare well to that of II-VI semiconductor-doped glasses and are much larger than that of colloidal melt-glasses.

For a metal cluster with a cubic symmetry, the fourth-rank tensor $\chi^{(3)}$ has four independent components. Of these, $\chi^{(3)} x x x x$ includes contributions from both thermal and electronic processes. The $\chi^{(3)} x x y y$ component, on the other hand, is sensitive only to electronic processes. The enhancement in the electronic component of $\chi^{(3)}$ due to the increased volume fraction 
$(\sim 30 \%)$ of the nanoclusters in the composite is obviously an important consideration from the point of view of non-linear optics, but achieving such a high concentration of dopants is a non-trivial materials problem. A volume fraction of such magnitude, obtainable by ion implantation only, cannot be made in a melt-glass because of the constraints of chemical and thermodynamic equilibrium. This points to one major advantage of ion-implantation as a technique for synthesizing nanocluster-glass composites.

\section{Magnitudes and temporal responses of $\chi(3)$ in nanocluster - glass composites}

Progress in non-linear optical materials is the key to creating functional photonic devices for optical communications, sensing and computing. Understanding the correlation between materials processing and optical nonlinearities is critical to the development of advanced non-linear materials for photonic devices. Optical switching at terahertz frequencies requires nonlinear optical materials with the following properties: picosecond or shorter response times, low-power switching threshold, high thermal stability, low two-photon absorption, wavelength tunability, and a high threshold for laser-induced damage.

There are many materials with high third-order nonlinearities, although only a large value of $\chi^{(3)}$ is not sufficient. The key issue for light-wave technology is to optimize performance of third-order materials in real device geometries under realistic operating conditions. Moreover, the long-term mechanical, thermal and optical stability of potential waveguide devices can only be tested accurately in actual device geometries. Thus the results achieved with metal-nanocluster glass composites made by ion implantation are significant because of their potential for making a high-performance nonlinear material using an established ion-implantation-based waveguide fabrication technology [64]. Figure (11) shows a comparison between a variety of electronic and photonic materials with respect to switching energy and switching speed [42].

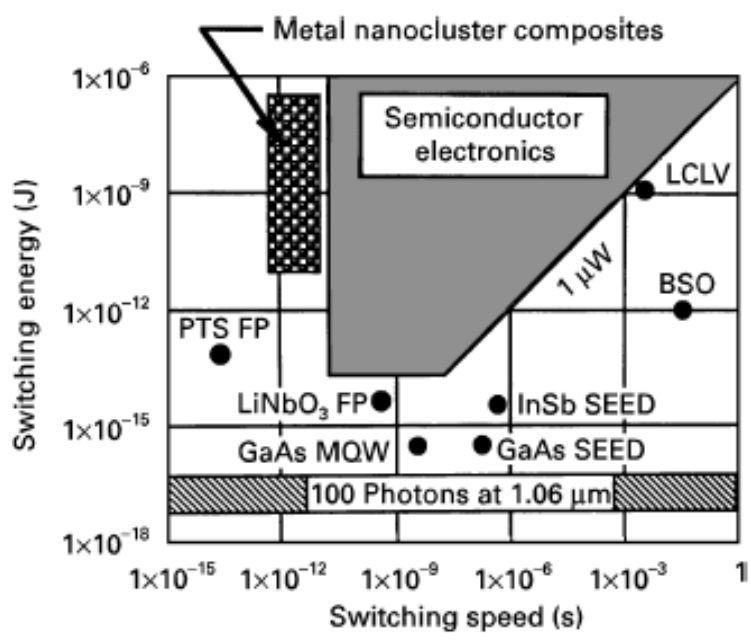

Fig. 11. Comparison of a variety of electronic and photonic materials with respect to switching energy and switching speed 
As shown in the hatched area, nanocluster composites of $\mathrm{Au}: \mathrm{SiO}_{2}$ and $\mathrm{Cu}: \mathrm{SiO}_{2}$ have faster switching speeds than any material, except para-toluene sulphonate (PTS), which, however, suffers from long-term photochemical damage. The shaded area in the figure indicates the range of switching energies projected from theoretical estimates for metal nanocluster composites. While no single material exhibits all the characteristics of an ideal optical switch, metal nanocluster composites do seem to offer an attractive combination. However, both for the demonstration of device potential and for studying optical physics in these interesting non-linear materials, it may be advantageous to carry out the non-linear studies in actual waveguide geometries. For example, because two-photon absorption, which is proportional to the imaginary part of $\chi^{(3)}$, arising not only from the clusters but also from the defects created by ion implantation, severely limits all-optical device performances, a fundamental understanding of its origin and relation to size, shape and density of the clusters in the waveguide is crucial. In optical switching devices and waveguide, a keyparameter is the non-linear index of refraction, proportional to the real part of $\chi^{(3)}$. Measurements of the magnitude and the temporal response characteristics of $\chi^{(3)}$ are the most important part of the experiments.

\subsection{Degenerate Four Wave Mixing (DFWM) \\ 5.1.1 Principle}

When a nonlinear material is illuminated by a pair of coherent waves of equal magnitude traveling in different directions, they result in a phase grating within the material, which in turn can diffract a third wave (not necessarily coherent with the first two) incident on the material into a fourth one. The diffracted phase conjugated wave ids the fourth wave. This process is called 'four-wave mixing' and has been demonstrated in several crystals. When all the four waves involved in the process have the same frequency, it is known as the degenerate four wave mixing (DFWM). The effect can be understood in terms of the third-order nonlinear susceptibility $\chi^{(3)}$. Physically, one may understand this process by considering the individual interactions of the fields within a dielectric medium. The first incident field causes in the dielectric an oscillating polarization, which re-radiates with some phase shift determined by the damping of the individual dipoles; this is just traditional Raleigh scattering described by linear optics. The application of a second field will also drive the polarization of the dielectric, and the interference of the two waves will cause harmonics in the polarization at the sum and difference frequencies. Now, the application of a third field will also drive the polarization at the sum and difference frequencies. This beating with the sum and difference frequencies is what gives rise to the fourth field in four wave mixing. Since each of the beat frequencies produced can also act as new source fields, a bewildering number of interactions and fields may be produced from this basic process.

\subsubsection{Theory}

The two coherent waves $\left(E=1 / 2 E_{0} \cos \omega t\right)$ with a time variation create an interference pattern or standing wave within the crystal. The pattern consists of regions near the nodes where the local field is essentially zero, and regions near the antinodes where its value is close to $E_{0} \cos \omega t$. Retaining terms up to the third order in polarization equation (Eq (2)), and adding a small extra field $\mathbf{E}^{\mathbf{E}}$ acting as the third field, we then have, up to the first order in $\mathbf{E}$ : 


$$
P+\delta P=\chi\left(E+E^{\prime}\right)+\chi^{(2)}\left(E^{2}+2 E E^{\prime}\right)+\chi^{(3)}\left(E^{3}+3 E^{2} E^{\prime}\right)
$$

Therefore,

$$
\delta P=\mathrm{E}^{\prime}\left(\chi+2 \chi^{(2)} E+3 \chi^{(3)} E^{2}\right)
$$

Now if $\mathbf{E}^{\prime}$ is incoherent with $\mathrm{E}$, the ratio $\delta P / \mathrm{E}^{\prime}$ is obtained by averaging the bracketed term in Equation (22) over many periods of $\omega$. The average of $E_{0} \cos \omega t$ of course is zero; that of $E_{0}^{2} \cos ^{2} \omega t$ is $E_{0}^{2} / 2$ and so, we have on average,

$$
\delta P / E^{\prime}=\left(\chi+3 / 2 \chi^{(3)} E_{0}^{2}\right)
$$

The refractive index seen by the field $\mathbf{E}^{\mathbf{}}$ is thus given by,

$$
\begin{gathered}
\mu_{d}=\left(1+\delta P / \varepsilon_{0} \mathrm{E}^{\prime}\right)^{1 / 2} \\
=\left[1+\left(\chi+3 / 2 \chi^{(3)} E_{0}^{2}\right) / \varepsilon_{0}\right]^{1 / 2}
\end{gathered}
$$

which depends on the field $\mathrm{E}_{0}$, and is therefore different at the nodes and the antinodes of the standing wave pattern created by the first two waves. The wave $\mathbf{E}^{\mathbf{v}}$, therefore, sees the standing wave 'written' into the crystal as a phase grating, which diffracts according to the Bragg's law. Figure (12) shows the forward and backward mixing arrangements of the DFWM setup [50]. In the former case there are two input beams, usually obtained by a mirror placed beyond the nonlinear medium. In both the cases, the nonlinear polarization radiates a signal field at $\omega$ in the phase-matched direction. The pump and the probe waves interfere giving rise to both amplitude gratings (resulting from the nonlinear loss due to two-photon absorption) and refractive index or phase gratings (due to the intensity- dependent refractive index change), from which the waves scatter coherently. The output intensities of signal and probe are related to the nonlinear absorption which can be related to the nonlinear refractive index.

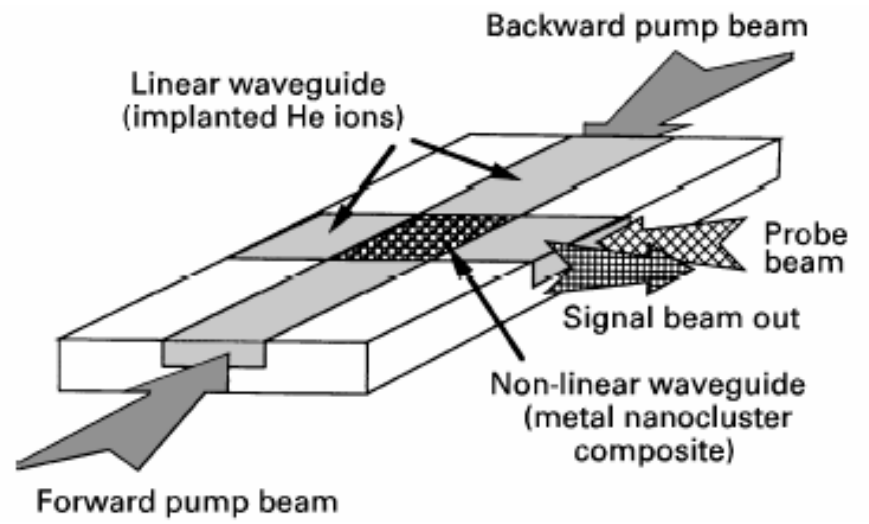

Fig. 12. The forward and backward mixing arrangements of the DFWM set up. 
Measurements of the magnitude and the temporal response characteristics of $\chi^{(3)}$ are the most important part of the experiments. One can measure $\chi^{(3)}$ at wavelengths both on and off the SPR by forward DFWM in the geometry of Fig. (12) [30]. In guided-wave geometry, it is possible to use a CW mode locked laser for four-wave mixing studies with counterpropagating pump-beams. In DFWM configuration, three different light fields, $E_{1}, E_{2}$ and $E_{3}$, of the same frequency are incident on the non-linear optical material to produce a fourth beam, $E_{4}$. It is also called phase conjugation or real-time holography. Two different types of volume holograms, i.e. reflection and transmission holograms can be constructed. In either type, the third beam is used as the reading beam and the fourth one is the result of the diffraction of the hologram, making $E_{4}$ conjugate to $E_{3}$. The third-order nonlinear optical susceptibility obtained from the above technique is given by [65]

$$
\chi^{(3)}=\frac{8 c^{2} n_{d}^{2} \varepsilon_{0} \alpha n^{1 / 2}}{3 \omega I_{1}(1-T)}
$$

where $n_{d}$ and $\alpha$ are linear refractive index of the host matrix and absorption coefficient of the nanocluster-glass composite, respectively, both measured at the same frequency, $\omega$, of the optical signal close to the SPR of the composite, $I_{1}$ is the pump intensity, $c$ is the velocity of light in vacuum, $\mathrm{T}$ is the transmittance of the medium at $\omega$, and $\mathrm{n}$ is the diffraction efficiency of the holographic grating. DFWM experiments also give important information on the switching speed. $\chi^{(3)}$ for gold nanoclusters in glass was measured by Magruder et al. [66] by DFWM using a mode-locked Q-switched, frequency doubled Nd:YAG laser at a wavelength of $532 \mathrm{~nm}$ with a pulse repetition frequency of $10 \mathrm{~Hz}$ and a nominal pulse width of $35 \pm 5$ ps. This wavelength is near the SPR of gold $(530 \mathrm{~nm})$. The two forward-going pumpbeams and a weak probe-beam were arranged to intersect in the composite-layer containing the metal clusters. The probe was delayed with respect to the two pump beams using a computer-controlled optical delay line. The pump and probe beams interacted coherently via the third-order non-linear susceptibility to produce a phase-conjugated signal, detected in a photomultiplier. Identical measurements were carried out on unimplanted samples; no measurable DFWM signal was observed. Figure (13) shows the intensity of the phase-

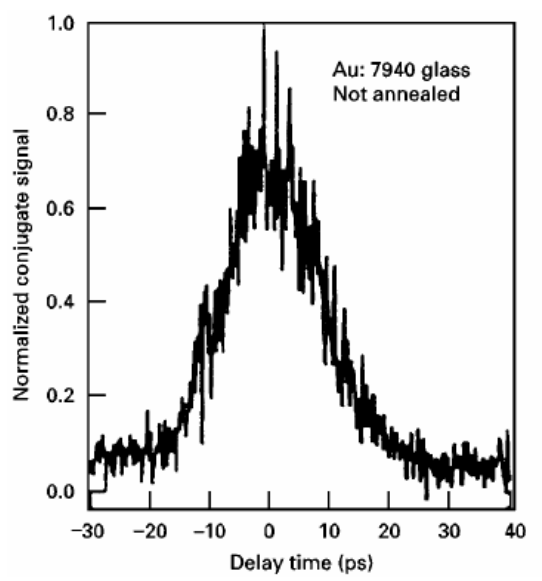

Fig. 13. Intensity of the phase-conjugated beam in a DFWM measurement with crosspolarized pump-probe configuration as a function of pump-probe time in picoseconds. 
conjugated signal as a function of pump probe delay time [66]. The symmetric shape of the time spectrum indicates that the third-order response is no longer than that of the pulse width ( $35 \mathrm{ps})$. The measured values of $\chi^{(3)}$ were found to be $1.0 \times 10^{-10}$ e.s.u and $1.7 \times 10^{-10}$ e.s.u. for this nanocluster composite layer without and with heat treatment, respectively.

\subsection{Z- Scan experiment}

\subsubsection{Principle}

Z-Scan technique [51], based on the principles of spatial beam distortions, allows us to determine both sign and magnitude of nonlinear refractive index $\eta_{2}$ and nonlinear absorption coefficient $\beta$ and it is very sensitive to detect small nonlinear refraction. The excitation source used in the Z-Scan measurement is a mode-locked laser. Each laser pulse has a Gaussian spatial profile and is focused by a converging lens. The sample is placed near the waist of a focused Gaussian beam and is scanned along the direction of propagation of the incident laser beam (Z-axis) of the light. As the sample is translated along Z-direction, it experiences a different incident field at different Z-positions. The light intensity transmitted across the nonlinear material is measured in the far field (FF) as the sample is moved along the direction of the propagation of light, in the open Z-Scan mode of operation. The transmittance of the nonlinear medium is measured through a finite aperture placed in the far field as a function of the sample position (Z). Figure (14) shows the schematics of a Z-scan set up. When the sample moves towards the focus from negative $Z$, the laser power density intercepted by the sample increases, giving rise to self-focusing [67]: owing to the Gaussian transverse intensity profile of the laser beam, the original plane wavefront gets progressively more disturbed, in a way similar to that imposed by a positive lens (in the case of positive nonlinearity of the material), leading to a self-focusing that shifts the position of the actual focal point. A photodiode detector simultaneously measures the intensity of the transmitted beam through the sample as a function of the position of the sample, giving the Z-Scan curve, the shape of which reveals the presence of any absorptive nonlinearity in the sample.

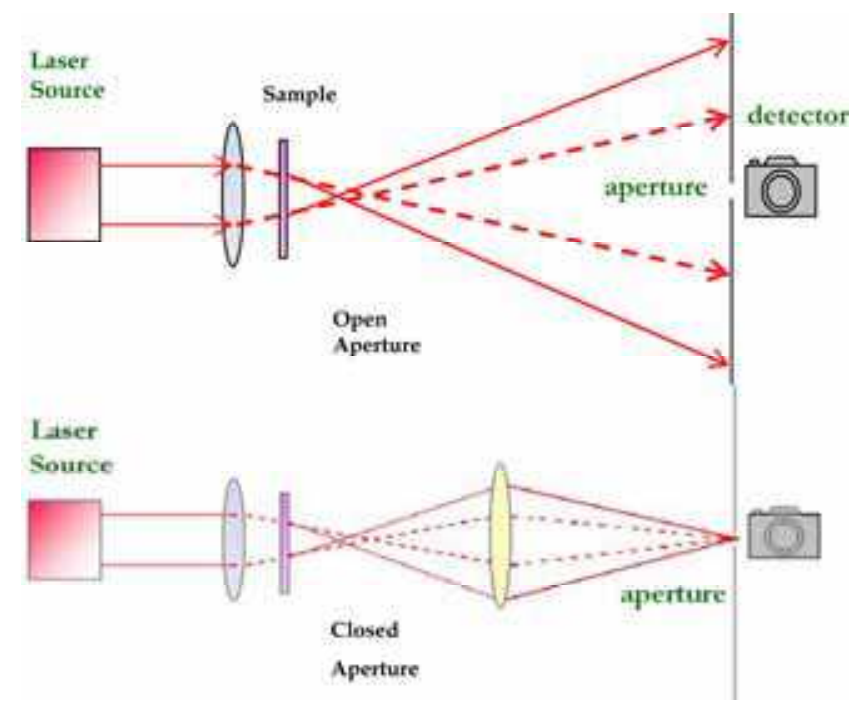

Fig. 14. Schematics of Z-scan experiment 
The sign of the nonlinear refraction is readily obtained from a Z-scan signature. An increase in transmittance in the pre-focal region followed by a decrease in the post-focal region (peak-valley) in the Z-scan signature denotes a negative nonlinear refraction, whereas a valley-peak configuration denotes a positive nonlinearity. Removal of the aperture, i.e., collecting all the light on detector, which is referred to as an open-aperture Z-scan, will result in a flat response for a purely refractive nonlinearity. It is because the sensitivity to nonlinear refraction is entirely due to the aperture, and its removal completely eliminates the effect. However, if nonlinear absorption is also present, then it will be reflected as a transmission variation in the open aperture scan. Multi-photon absorption suppresses peak and enhances the valley while saturation of absorption produces the opposite effect in a closed-aperture Z-scan. Thus, apart from the magnitude of the nonlinearity (both real and imaginary parts), this technique provides a direct measurement of the sign of nonlinearity also. The sign of the nonlinearity is an important parameter for practical realization of optical signal processing devices.

\subsubsection{Theory}

When a matter is subjected to an intense laser radiation, it exhibits intensity-dependent index of refraction given by

$$
n(I)=n_{0}+n_{2} I
$$

That is,

$$
n(I)=n_{0}+\Delta n(I)
$$

where $n_{0}$ is the linear refractive index and $\Delta n(I) \equiv n_{2} I$ is the intensity dependent change in the refractive index. $n_{2}$ is called the refractive index intensity coefficient and is related to $\chi^{(3)}$ by

$$
\chi^{(3)}(e s u)=\frac{n_{0}^{2}}{4 \pi^{2}} 10^{-6} n_{2}
$$

where $n_{2}$ is expressed in $\mathrm{cm}^{2} / \mathrm{GW}$.

Consequently, the phase change of the intense laser beam on propagation through a distance $L$ through the nonlinear medium will be

$$
\begin{aligned}
\Phi & =k n(I) L \\
& =K\left(n_{0}+\Delta n(I)\right) L \\
& =\Phi^{L}+\Phi^{N L}
\end{aligned}
$$

In the above expression, the first term is the linear phase shift. The second term is the nonlinear phase shift arising from nonlinear refraction and can be written as

$$
\begin{gathered}
\Phi^{N L}=k(\Delta n) L \\
=k n_{2} I L
\end{gathered}
$$

It is thus clear that due to effect of $n_{2}$, the beam passing through a nonlinear optical medium has an additional phase shift which is intensity dependent. Consequently, after 
passing through a nonlinear medium, the beam wavefront is modified due to intensitydependent phase changes. In Z-scan technique, these nonlinear phase front distortions are translated into a transmission variation of an aperture placed in the far field. Hence the transmittance of the aperture is a measure of the nonlinear phase change or $n_{2}$ and consequently, $\chi^{(3)}$.

The electric field of a Gaussian beam having waist radius $w_{0}$ and traveling in the $+Z$ direction is represented as

$$
E(Z, r, t)=E_{0}(t) \frac{w_{0}}{w(Z)} \exp \left(-\frac{r^{2}}{w^{2}(Z)}-\frac{i k r^{2}}{2 R(Z)}\right) e^{-i \phi(Z, t)}
$$

where

$$
w^{2}(Z)=w_{0}^{2}\left(1+\frac{Z^{2}}{Z_{0}^{2}}\right)
$$

is the beam cross-section at $Z$,

$$
R(Z)=Z\left(1+\frac{Z_{0}^{2}}{Z^{2}}\right)
$$

is the radius of curvature of the wavefront at $Z$,

$$
Z_{0}=\frac{\pi w_{0}^{2}}{\lambda}
$$

is the Rayleigh range,

$$
k=\frac{2 \pi}{\lambda}
$$

is the wave vector, and $\lambda$ is the laser wavelength, all in free space. $E_{0}(t)$ denotes the radiation electric field at the focus and contains the temporal envelope of the laser pulse. The exponential term $\exp \{-i \phi(Z, t)\}$ represents the radial phase variations. If the sample length is small enough such that the changes in the beam diameter within the sample due to either diffraction or nonlinear refraction can be neglected, the sample is regarded as thin. Considering a thin sample and using the slowly varying envelope approximation, the wave equation for the phase and intensity can be respectively written as

$$
\begin{gathered}
\frac{d \Delta \phi}{d z^{\prime}}=\Delta n(I) k \\
\frac{d I}{d z^{\prime}}=-\alpha(I) I
\end{gathered}
$$

where $z^{\prime}$ is the propagation distance inside the sample and $\alpha(I)$, in general, includes the linear and nonlinear absorption terms. For cubic nonlinearity and negligible absorption, Eqs. 
(32) and (33) are solved to give the phase shift $\Delta \phi$ at the exit surface of the sample, which simply follows the radial variation of the incident irradiance at a given position of the sample Z :

$$
\Delta \phi(Z, r, t)=\Delta \phi_{0}(Z, t) \exp \left(-\frac{2 r^{2}}{w^{2}(Z)}\right)
$$

with

$$
\Delta \phi_{0}(Z, t)=\frac{\Delta \phi_{0}(t)}{1+Z^{2} / Z_{0}^{2}}
$$

where $\Delta \phi_{0}(t)$ is the on-axis phase shift at focus and is defined as

$$
\Delta \phi_{0}(t)=k \Delta n_{0}(t) L_{e f f}
$$

where

$$
L_{e f f}=\frac{1-\exp (-\alpha l)}{\alpha}
$$

and

$$
\Delta n_{0}=n_{2} I_{0}(t)
$$

Here $\alpha$ is the linear absorption coefficient of the sample, $l$ is the sample length, $n_{2}$ is the intensity dependent refractive index and $I_{0}(t)$ is the on-axis irradiance at focus (i.e., at $Z=0$ ). The complex electric field exiting the sample $E_{e}$ will contain the nonlinear phase distortion and can be represented as

$$
E_{e}(Z, r, t)=E(Z, r, t) \exp (-\alpha L / 2) \exp \{i \Delta \phi(Z, r, t)\}
$$

Now, applying the Gaussian beam decomposition method, we decompose the complex electric field at the exit plane of the sample given by Eq. (40) into a summation of Gaussian beams through a Taylor series expansion of the nonlinear phase term $\exp \{i \Delta \phi(Z, r, t)\}$. That is,

$$
\exp \{i \Delta \phi(Z, r, t)\}=\sum_{m=0}^{\infty} \frac{\left[i \Delta \phi_{0}(Z, t)\right]^{m}}{m !} \exp \left[-\frac{2 m r^{2}}{w^{2}(Z)}\right]
$$

Now each Gaussian beam can be simply propagated to the aperture plane where they are reassumed to reconstruct the beam. The resultant electric field at the aperture plane is given by

$$
\begin{aligned}
E_{a}(r, t)=E(Z, r= & 0, t) \exp (-\alpha L / 2) \sum_{m=0}^{\infty} \frac{\left[i \Delta \phi_{0}(Z, t)\right]^{m}}{m !} \frac{w_{m 0}}{w_{m}} \\
& \exp \left(-\frac{r^{2}}{w_{m}^{2}}-\frac{i k r^{2}}{2 R_{m}}+i \theta_{m}\right)
\end{aligned}
$$


where we have used the following abbreviations:

$$
\begin{gathered}
w_{m 0}^{2}=\frac{w^{2}(Z)}{2 m+1} \\
w_{m}^{2}=w_{m 0}^{2}\left[g^{2}+\frac{d^{2}}{d_{m}^{2}}\right] \\
g=1+\frac{d}{R(Z)} \\
d_{m}=\frac{k w_{m 0}^{2}}{2} \\
R_{m}=d\left[1-\frac{g}{g^{2}+d^{2} / d_{m}^{2}}\right]^{-1}
\end{gathered}
$$

and

$$
\theta_{m}=\tan ^{-1}\left[\frac{d / d_{m}}{g}\right]
$$

Here $\mathrm{d}$ is the propagation distance from the sample to the aperture. The transmitted power through the aperture is obtained by spatially integrating $E_{a}(r, t)$ over the aperture radius $r_{a}$, giving

$$
P_{T}(t)=c \varepsilon_{0} \pi \int_{0}^{r_{a}}\left|E_{a}(r, t)\right|^{2} r d r
$$

Including the pulse temporal variation, the normalized $Z$-scan transmittance $T(Z)$ can be calculated as

$$
T(Z)=\frac{\int_{-\infty}^{\infty} P_{T}(t) d t}{S \int_{-\infty}^{\infty} P_{i}(t) d t}
$$

where

$$
P_{i}(t)=\frac{\pi w_{0}^{2} I_{0}(t)}{2}
$$

is the instantaneous input power and

$$
S=1-\exp \left(-2 r_{a}^{2} / w_{a}^{2}\right)
$$


is the aperture linear transmittance, with $w_{a}$ denoting the beam radius at the aperture in the linear regime. In case the sample exhibits nonlinear absorption, open aperture scan will reflect that, while closed aperture scan records coupled refractive and absorptive parts. Therefore, one has to deduce the nonlinear absorption coefficient from open aperture scan, and then using closed aperture, the refractive and absorptive nonlinearity can be separated out. For example, if the nonlinear absorption is of two-photon in nature, then the absorption coefficient is given by

$$
\alpha(I)=\alpha_{0}+\beta I
$$

The phase shift and irradiance distribution at the exit surface of the sample as

$$
I_{e}(Z, r, t)=\frac{I(Z, r, t) e^{-\alpha L}}{1+q(Z, r, t)}
$$

and

$$
\Delta \phi(Z, r, t)=\frac{k n_{2}}{\beta} \ln [1+q(Z, r, t)]
$$

where $q(Z, r, t)=\beta I(Z, r, t) L_{\text {eff }}$. Combining Eqs. (46) and (47), the complex electric field at the exit surface of the sample is given as

$$
E_{e}(Z, r, t)=E(Z, r, t) e^{-\alpha L} / 2(1+q)^{\left(i k n_{2} / \beta-1 / 2\right)}
$$

We can see that Eq. (48) reduces to (39) in the limit of no nonlinear absorption (i.e. $\beta=0$ ). For $|q|<1$, following a binomial series expansion in powers of q, Eq. (48) can be expressed as an infinite sum of Gaussian beams similar to the purely refractive case described above as follows:

$$
\begin{array}{r}
E_{e}(Z, r, t)=E(Z, r, t) e^{-\alpha L / 2} \sum_{m=0}^{\infty} \frac{q(Z, r, t)^{m}}{m !} \\
{\left[\prod_{n=0}\left(i k n_{2} / \beta-1 / 2-n+1\right)\right]}
\end{array}
$$

Now we can write the complex field pattern at the aperture plane in the same manner as described in purely refractive case provided the phase term in Eq. (40) is replaced by

$$
f_{m}=\frac{\left(i \Delta \phi_{0}(z, t)\right)^{m}}{m !} \prod_{n=0}^{m}\left(1+i(2 n-1) \frac{\beta}{2 k n_{2}}\right)
$$

The normalized transmittance can now be estimated following the same procedure as described in purely absorptive case. It is evident from Eq. (50) that the absorptive and refractive contributions to the far-field beam profile and hence to the $Z$-scan transmittance are coupled to each other due to the coupling factor $\beta / 2 k n_{2}$ which is the ratio of the 
imaginary to the real parts of the third-order nonlinear susceptibility $\chi^{(3)}$. When the aperture is removed, the $Z$-scan transmittance is insensitive to the beam distortion and is only a function of the nonlinear absorption. The total transmitted power in that case $(S=1)$ is obtained by spatially integrating Eq. (46), which yields

$$
P(Z, t)=P_{i}(t) e^{-\alpha L} \frac{\ln \left[1+q_{0}(Z, t)\right]}{q_{0}(Z, t)}
$$

where

$$
q_{0}(Z, t)=\frac{\beta I_{0}(t) L_{e f f}}{1+\frac{Z^{2}}{Z_{0}^{2}}}
$$

For a temporally Gaussian pulse, Eq. (51) can be time-integrated to give the normalized energy transmittance as

$$
T(Z, S=1)=\frac{1}{\sqrt{\pi} q_{0}(Z, 0)} \int_{-\infty}^{\infty} \ln \left[1+q_{0}(Z, 0) e^{-\tau^{2}}\right] d \tau
$$

For $\left|q_{0}\right|<1$, this energy transmittance can be expressed in terms of the peak irradiance in a summation form as

$$
T(Z, S=1)=\sum_{m=0}^{\infty} \frac{\left[-q_{0}(Z, 0)\right]^{m}}{(m+1)^{3 / 2}}
$$

Thus, once an open aperture $(S=1) Z$-scan is performed, the nonlinear absorption coefficient $\beta$ can be estimated using Eq. (54). With $\beta$ known, the $Z$-scan with aperture in place $(S<1)$ can be used to extract the nonlinear refractive index coefficient $\eta_{2}$. Similarly in case of other nonlinearities (e.g. saturation of absorption, excited state absorption, free carrier absorption etc.), we can deduce the refractive and absorptive contribution to nonlinearity by modifying the equations for phase shift and beam attenuation, given by Eqs. (33) and (34) respectively, and solving for nonlinear phase shift and field amplitude at the exit surface of the sample.

Sheik et. al. [51] have shown that the above tedious calculations can be simplified provided the phase change induced by the nonlinear medium is small. For $|\Delta \phi|<\pi$, the peak-valley difference in transmission in case of purely refractive nonlinearity is given by

$$
T_{p-v}=0.405(1-S)^{0.25} k n_{2} I_{0} L_{e f f}
$$

which is valid within $3 \%$. The peak-valley separation along $Z$-axis in this case is related to Rayleigh range through

$$
Z_{p-v}=1.7 Z_{0}
$$

Therefore, we can get quick estimate of nonlinearity by just measuring the peak-valley difference in transmission. 


\subsubsection{Z-scan simulations}

Figure (15) shows open aperture $Z$-scan simulations for positive and negative $\beta$ respectively. In case of positive $\beta$ (two-photon absorption, excited state absorption or freecarrier absorption), we get a valley at the beam waist position of the focused beam while negative $\beta$ (saturation of absorption) results in a peak at the same position. Thus, the sign of absorptive nonlinearity is readily obtained from the open aperture $Z$-scan signature. If the sample does not exhibit any nonlinear absorption, then no transmittance variation will be recorded in the open-aperture

Figure (16) shows $Z$-scan simulation for purely refractive positive and negative nonlinearities corresponding to a phase change $\Delta \phi= \pm 0.5$ and an aperture parameter $S=0.2$. As explained earlier, negative nonlinear refraction results in increase in transmittance in the pre-focal region followed by decrease in the post-focal region (peakvalley) while positive nonlinear refraction exhibits the opposite behaviour. Also shown is the open aperture simulation in which there is no transmittance variation in case of purely refractive nonlinearity.
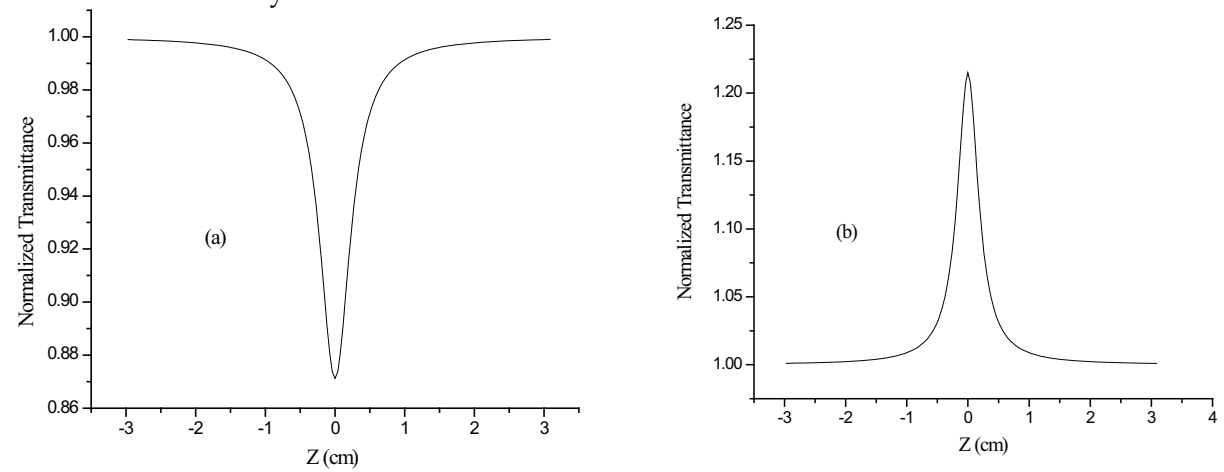

Fig. 15. Open aperture Z-scan simulations for (a) positive $\beta$ and (b) negative $\beta$
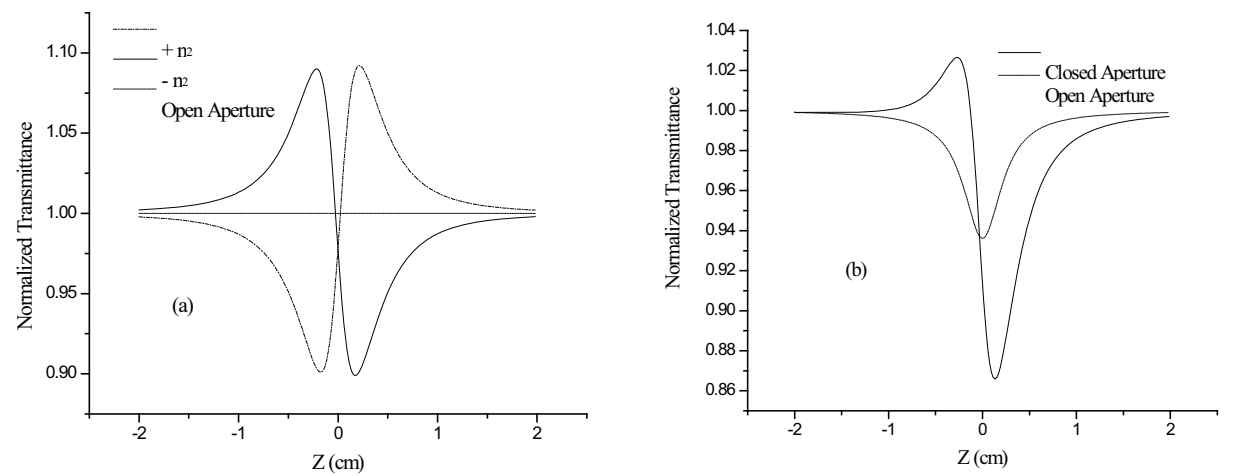

Fig. 16. Z-scan simulations for (a) purely refractive and (b) refractive + absorptive nonlinearity.

When nonlinear absorption is also present, then in the closed aperture signature, the peak gets suppressed while the valley gets enhanced for positive absorptive nonlinearity. The opposite behaviour will be exhibited in case of negative absorptive nonlinearity. Fig. shows 
simulation for a negative refractive nonlinearity and positive absorptive nonlinearity for a phase change of $\Delta \phi=-0.5$ and two-photon absorption parameter $q_{0}=0.2$.

\subsection{Anti-Resonant Ring Interferometric Nonlinear Spectroscopy (ARINS)}

\subsubsection{Principle}

Lee and Hughes (LH) [68] proposed a simple, sensitive, single beam technique based on an anti-resonant ring (Sagnac) interferometer for simultaneously measuring the real and imaginary contributions to optical nonlinearity, and called it Anti-resonant Interferometric Nonlinear Spectroscopy (ARINS). The ARINS technique utilizes the dressing of two unequal-intensity counter-propagating pulsed beams with differential nonlinear phases, which occurs upon traversing the sample. This difference in phase manifests itself in the intensity dependent transmission. Photo-detection of the transmission of the ARINS yields spatially and temporally integrated response. Consequently, it is the pulse energy and not the instantaneous power that is detected. Figure (17) shows the schematic diagram of ARINS setup [69].

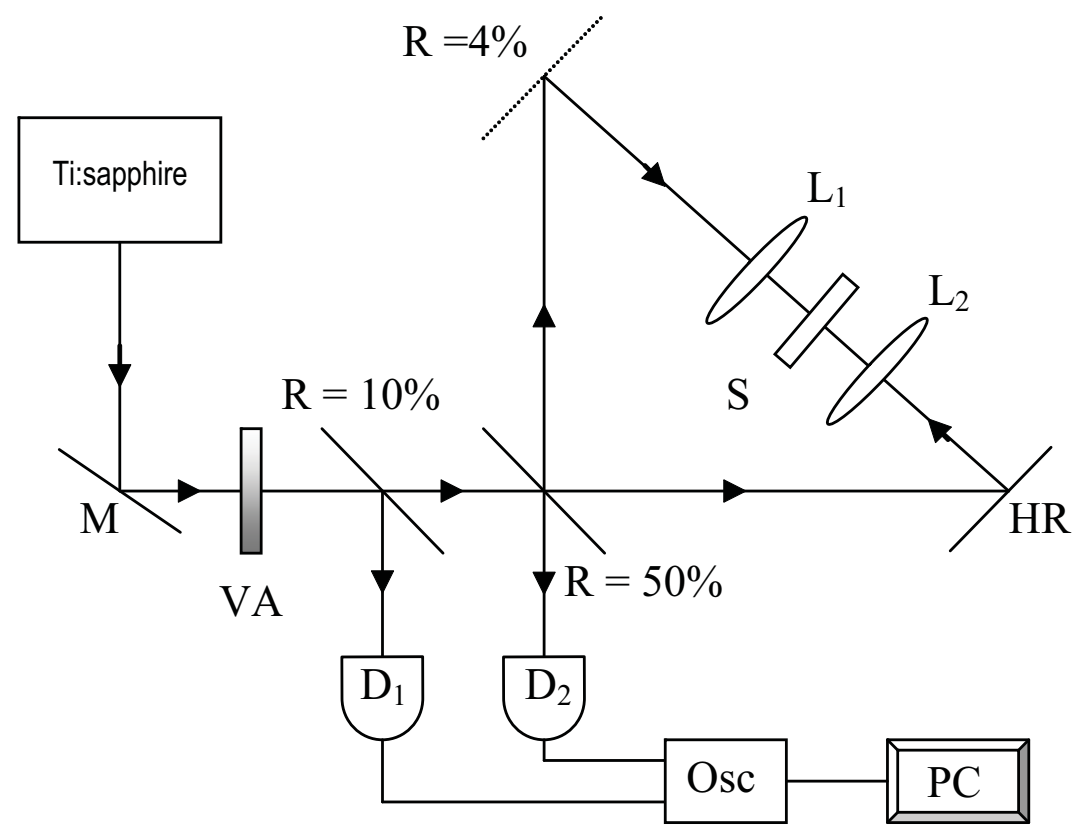

Fig. 17. ARINS experimental setup. M- mirror, L - lenses, HR - high reflectivity mirror, $\mathrm{S}$ sample, VA - variable attenuator D- detectors, Osc - digital oscilloscope, PC microcomputer

A 50-50 beam splitter divides the incoming pulsed beam into two counter-propagating pulses having a $\pi$ phase difference. The pulses propagating in the clockwise (CW) direction are reflected by an uncoated flat with $6^{\circ}$ wedged rear surface while that propagating in the counterclockwise $(\mathrm{CCW})$ direction are reflected by a high reflectivity mirror. Thus this technique is based on the dressing of two unequal intensity counter-propagating pulses. 
These two pulses again recombine at the beam splitter to yield ARINS transmission, $\left|E_{\text {out }}\right|^{2} \propto\left|E_{c w}+E_{c c w}\right|^{2}$, where $E_{c w}$ and $E_{c c w}$ are the optical fields travelling in clockwise and counterclockwise directions respectively. Inside the ring there is a unit magnification telescope comprising of a pair of identical lenses in $2 f$ configuration. One of the lenses focuses the pulses into the sample placed at its focus while the other one re-collimates them. The two counter propagating fields traversing the ring acquires linear as well as intensity dependent nonlinear phase shifts (if the sample exhibits nonlinear response). Since both the fields traverse the same optical path through the ring and encounter the same interactions with the optical elements, linear interactions would affect their amplitude and phase in identical manner. For an exactly $50 \%$ beam splitter, in the absence of any nonlinear interactions, the two returning fields will be having the same amplitude and phase difference between them and consequently they will interfere destructively at the beam splitter to yield zero transmission. In this 'balanced' condition all the input power is reflected back to the incident direction. Measurement against this dark background provides the basis for the improved sensitivity essential for measuring relatively weak signals. Any small deviation from the ideal splitting ratio $(\delta)$ results in a leakage from the ARINS and is responsible for the background that limits the sensitivity of the measurement.

If the sample under investigation exhibits nonlinear response, then the two unequalintensity counter-propagating pulses will undergo different phase changes after passing through the sample. Their superposition on the beam splitter will result in the intensity dependent transmission of the ARINS, which is related to the nonlinear response of the sample. However, differential dressing of the two counter propagating pulses with nonlinear phases is possible only when the two do not interact simultaneously in the sample. Else, the same nonlinear phase will be impressed on both by the cross-action phenomenon. Therefore, we prevent the temporal overlap of the two pulses in the sample by spatially offsetting it with respect to the center of the ARINS in such a way that the intense beam (i.e. CCW beam in our geometry) should reach the sample first in order to initiate nonlinear processes. The time difference between the arrivals of the two pulses $\left(\Delta \tau_{\text {arr }}\right)$ determines the nature of the nonlinear optical process that can be studied depending on its response time. Nonlinear processes with decay time longer that $\Delta \tau_{a r r}$ do not contribute to the intensity dependent transmission of the ARINS as both the pulses will be affected simultaneously in that case. The delay window thus acts as an ultrafast gate. Hence this technique has the unique ability to filter the nonresonant electronic contributions from integrating (or slow, e.g. thermal) nonlinearities or those arising from long lived (resonant) states and makes it ideal for time-resolved studies and ultrafast gating. If the arrival times of the two pulses in the sample were reversed, one would measure the unfiltered response.

\subsubsection{Theory}

Equation Chapter 2 Section 1Let us consider a collimated, spatially and temporally coherent Gaussian pulse with electric field amplitude $E_{0}$, incident on the beam splitter, where it splits into two counter-propagating beams with electric fields $E_{c w}$ and $E_{c c w}$. The general form for the electric field of a Gaussian beam is given by 


$$
E(Z, r, t)=E_{0} \frac{w_{0}}{w(Z)} \exp \left[\frac{-r^{2}}{w^{2}(Z)}\right] \exp [i \phi(Z)] F(t)
$$

where $\mathrm{Z}$ is the distance of propagation, $\mathrm{r}$ is the transverse coordinate, $w_{0}$ is the beam waist $(Z=0), \phi(Z)=\exp \left[i\left(k Z-\tan ^{-1}\left(Z / Z_{0}\right)\right)\right]$ is the phase of the Gaussian beam (with pulse duration $\tau_{p}$ and wave vector $\mathrm{k}$ ) and the function $F(t)=\exp \left[(-2 \ln 2) t^{2} / \tau_{p}^{2}\right]$ gives the temporal variation of the pulse. The spot size at a distance $Z$ is $w^{2}(Z)=w_{0}^{2}\left[1+\left(Z^{2} / Z_{0}^{2}\right)\right]$, where $Z_{0}$ is the Rayleigh range. For simplicity, we assume that the Rayleigh range is larger than the sample thickness (thin sample approximation). We ignore the slight difference in the spot sizes for the CW and CCW beams at the lenses that arises because the sample is offset from the center of the ARINS. The intensity ratios in CW and CCW directions are $(1 / 2-\delta)$ and $(1 / 2+\delta)$ respectively, where, as said earlier, $\delta$ represents the small deviation from the ideal splitting ratio of the beam splitter. The electric field at the incident face for the two counter propagating beams can then be expressed as [70]

$$
\begin{gathered}
E_{c w}=-\sqrt{1 / 2-\delta} E_{0} \exp \left(-r^{2} / w_{0}^{2}\right) F(t) \sqrt{R} \\
E_{c w}=\sqrt{1 / 2+\delta} E_{0} \exp \left(-r^{2} / w_{0}^{2}\right) F(t)
\end{gathered}
$$

where $\mathrm{R}$ is the reflectivity of the uncoated flat. For the weaker $\mathrm{CW}$ beam, $\alpha(I)=\alpha$, the coefficient of linear absorption and $n(I)=n_{0}$, the linear refractive index. However, for the stronger CCW beam, $\alpha(I)=\alpha+\beta I$ and $n(I)=n_{0}+n_{2} I$, where $\beta$ is the effective nonlinear absorption coefficient and $n_{2}$ is the nonlinear index of refraction. The electric field of a pulsed Gaussian beam at the exit face of the sample (thickness $=$ L) with a nonlinear absorption and nonlinear refractive index is

$$
E_{\text {exit }}(r, t)=\frac{E_{0}(r, t)}{\sqrt{1+q}} \exp (-\alpha L / 2) \exp \left(-i k n_{0} L\right) \exp \left(-i k n_{2} \ln (1+q) / \beta\right)
$$

where $E_{0}(r, t)$ is the incident electric field, $q=\beta I_{\text {in }} L_{\text {eff }}, L_{\text {eff }}=[1-\exp (-\alpha L)] / \alpha$ is the effective length of the sample, $I_{0}$ is the intensity incident on the beam splitter, $I_{\text {in }}=(1 / 2+\delta) K^{\prime} I_{0}=K I_{0}$ is the intensity incident on the sample, $K^{\prime}$ is a constant $(<1)$ accounting for the reflection losses at the sample and lens surfaces while $K=(1 / 2+\delta) K^{\prime}$ is another constant. Using Eqs. (57), (58) and (59), the electric field of the two counterpropagating beams at the corresponding exit faces of the sample can be written as 


$$
\begin{gathered}
E_{c w}^{e x i t}=-\sqrt{(1 / 2-\delta)} E_{0} \exp \left(\frac{-r^{2}}{w_{0}^{2}}\right) \exp (-\alpha L / 2) \exp \left(-i k n_{0} L\right) F(t) \sqrt{R} \\
E_{c c w}^{e x i t}=\sqrt{(1 / 2+\delta)} \frac{E_{0}}{\sqrt{1+q}} \exp \left(\frac{-r^{2}}{w_{0}^{2}}\right) \exp (-\alpha L / 2) \exp \left(-i k n_{0} L\right) \exp \left(-i k n_{2} I_{i n} L_{\text {eff }}\right) F(t)
\end{gathered}
$$

When the two beams arrive again at the beam splitter after one trip round the ring, the electric fields in the transmission branch are given by multiplying Eqs. (60) and (61) by the respective splitting ratios

$$
\begin{gathered}
E_{c w}^{t}=-(1 / 2-\delta) E_{0} \frac{w_{0}}{w(Z)} \exp \left(\frac{-r^{2}}{w^{2}(Z)}\right) \exp (-\alpha L / 2) \exp \left(-i k n_{0} L\right) \exp [i \phi(Z)] F(t) \sqrt{R} \\
E_{c c w}^{t}=(1 / 2+\delta) \frac{E_{0}}{\sqrt{1+q}} \frac{w_{0}}{w(Z)} \exp \left(\frac{-r^{2}}{w^{2}(Z)}\right) \exp (-\alpha L / 2) \exp \left(-i k n_{0} L\right) \exp [i \phi(Z)] \\
\quad \times \exp \left(-i k n_{2} I_{i n} L_{\text {eff }}\right) F(t) \sqrt{R}
\end{gathered}
$$

where $w(Z)$ is the spot size at the beam splitter after one round trip. The values of $w(Z)$ and $w_{0}$ can be determined experimentally. The ARINS leakage is given by

$$
\left|E_{\text {out }}(r, t)\right|^{2}=\left|E_{c w}^{t}(r, t)+E_{c c w}^{t}(r, t)\right|^{2}
$$

Substituting Eqs. (62) and (63) in Eq. (64), we get

$$
\begin{aligned}
\left|E_{\text {out }}\right|^{2}= & {\left[\frac{w_{0}^{2}}{w^{2}(Z)} \exp (-\alpha L) \exp \left(\frac{-2 r^{2}}{w^{2}(Z)}\right) F^{2}(t)\right] } \\
\times & {\left[\left(\frac{1}{2}-\delta\right)^{2}+\frac{(1 / 2+\delta)^{2}}{1+q}+\left(2 \delta^{2}-\frac{1}{2}\right) \cos \left(k n_{2} I_{\text {in }} L_{\text {eff }}\right)\right]\left|E_{0}\right|^{2} R }
\end{aligned}
$$

For $q<<1$, Eq. (65) becomes

$$
\begin{aligned}
\left|E_{\text {out }}\right|^{2}= & {\left[\frac{w_{0}^{2}}{w^{2}(Z)} \exp (-\alpha L) \exp \left(\frac{-2 r^{2}}{w^{2}(Z)}\right) F^{2}(t)\right] } \\
\times & {\left[4 \delta^{2}+\beta I_{\text {in }} L \delta+\left[\left(\frac{\beta I_{\text {in }}}{4}\right)^{2}+\left(\frac{k n_{2} I_{\text {in }}}{2}\right)^{2}\right] L^{2}\right]\left|E_{0}\right|^{2} R }
\end{aligned}
$$

The relevant measured quantity in this experiment is the transmitted pulse energy: 


$$
W=2 n c \varepsilon_{0} \int_{-\infty}^{\infty} \int_{0}^{\infty} E_{\text {out }}^{2} 2 \pi r d r d t
$$

Using Eq. (66), and carrying out the integration in Eq. (67), we get

$$
\begin{aligned}
W=2 n c \varepsilon_{0} & \left(\frac{\pi \sqrt{\pi} w^{2}(Z) R \tau I_{0} \exp (-\alpha L)}{2 \sqrt{\ln 2}}\right) \\
\times & {\left[4 \delta^{2}+\frac{\beta L \delta I_{i n}}{2 \sqrt{2}}+\left[\left(\frac{\beta}{4}\right)^{2}+\left(\frac{k n_{2}}{2}\right)^{2}\right] \frac{L^{2} I_{i n}^{2}}{3 \sqrt{3}}\right] }
\end{aligned}
$$

Defining

$$
I_{\text {out }}=2(\ln 2)^{1 / 2} W / \pi^{3 / 2} \tau w^{2}(Z)
$$

which has the dimensions of intensity, Eq. (68) can now be expressed as

$$
I_{\text {out }}=2 n c \varepsilon_{0} R I_{0} \exp (-\alpha L) \times\left[4 \delta^{2}+\frac{\beta L \delta I_{\text {in }}}{2 \sqrt{2}}+\left[\left(\frac{\beta}{4}\right)^{2}+\left(\frac{k n_{2}}{2}\right)^{2}\right] \frac{L^{2} I_{\text {in }}^{2}}{3 \sqrt{3}}\right]
$$

Using $I_{\text {in }}=K I_{0}$ as explained earlier, we get

$$
I_{\text {out }}=2 n c \varepsilon_{0} R \exp (-\alpha L) \times\left[4 \delta^{2} I_{0}+\frac{\beta L \delta K}{2 \sqrt{2}} I_{0}^{2}+\left[\left(\frac{\beta}{4}\right)^{2}+\left(\frac{k n_{2}}{2}\right)^{2}\right] \frac{L^{2} K^{2}}{3 \sqrt{3}} I_{0}^{3}\right]
$$

For low intensity levels $q<<1$, the ARINS transmission $I_{\text {out }}$ as a function of $I_{\text {in }}$ is a cubic polynomial [70]. The coefficients are directly related to the coefficient of nonlinear refractive index, $n_{2}$ and to the coefficient of nonlinear absorption, $\beta$ (which may be due to 'saturation of absorption' or 'two-photon absorption' or 'excited-state absorption'). As stated earlier, $\delta$ is related to the linear leakage and limits the sensitivity. For $\delta=0, I_{\text {out }} \propto I_{\text {in }}^{3}$, in which case the simultaneous evaluation of $n_{2}$ and $\beta$ becomes difficult. However, a small nonzero $\delta$ makes the analysis much simpler as each term of the polynomial can be evaluated directly. If $\delta>0$ and the samples exhibits two-photon absorption or excited-state absorption (i.e. $\beta>0$ ), then all the coefficients of the polynomial become positive and $I_{\text {out }}$ will be a continuously increasing function of $I_{\text {in }}$. On the other hand, if the sample exhibits saturation of absorption (i.e. $\beta<0$ ) then the sign of $\beta$ opposes the increase in nonlinear leakage. In this case, the quadratic term in the polynomial becomes negative and $I_{\text {out }}$ shows saturation at a relatively low intensity, where the effect of $\delta$ is cancelled by the saturable absorption coefficient, $\beta$. However, at higher intensities, the cubic term becomes dominant and $I_{\text {out }}$ starts increasing continuously. For $\delta<0$, the curvature of ARINS leakage as a function of $I_{\text {in }}$ is reversed for the two processes. Thus, by carefully choosing the value of $\delta$, both the origin of nonlinearity and the values of nonlinear coefficients can be conveniently obtained. 


\subsubsection{ARINS simulations}

The calculated ARINS transmission using Eq. (70) with typical values of $n_{2}=1.8 \times 10^{-6} \mathrm{~cm}^{2} / \mathrm{GW}$ and $\beta=0.378 \mathrm{~cm} / \mathrm{GW}$ by choosing different values of $\delta$ is shown in Figure (18).

The above simulation shows that the ARINS leakage is very sensitive to both sign and magnitude of $\delta$. In fact, it is the combination of both $\delta$ and $\beta$ which determines the sign and magnitude of the quadratic term in Eq. (70). If the sample exhibits nonlinear absorption (not saturation) then $\beta>0$. In this case, for $\delta>0$, all the coefficients of the polynomial will be positive and $\mathrm{I}_{\text {out }}$ will be a continuously increasing function of $\mathrm{I}_{\mathrm{in}}$. However, if the sample exhibits saturation of absorption $(\beta<0)$, then the quadratic term in Eq. (70) becomes negative and $\mathrm{I}_{\text {out }}$ shows saturation at a relatively low intensity where the effect of $\delta$ is cancelled by the saturable absorption. Thus the curvature experiences a point of inflexion in this case. At higher intensities, the cubic term in Eq. (70) dominates and therefore $\mathrm{I}_{\text {out }}$ increases continuously. For $\delta<0$, the curvature of ARINS leakage as a function of $\mathrm{I}_{\text {in }}$ is reversed for the two processes.
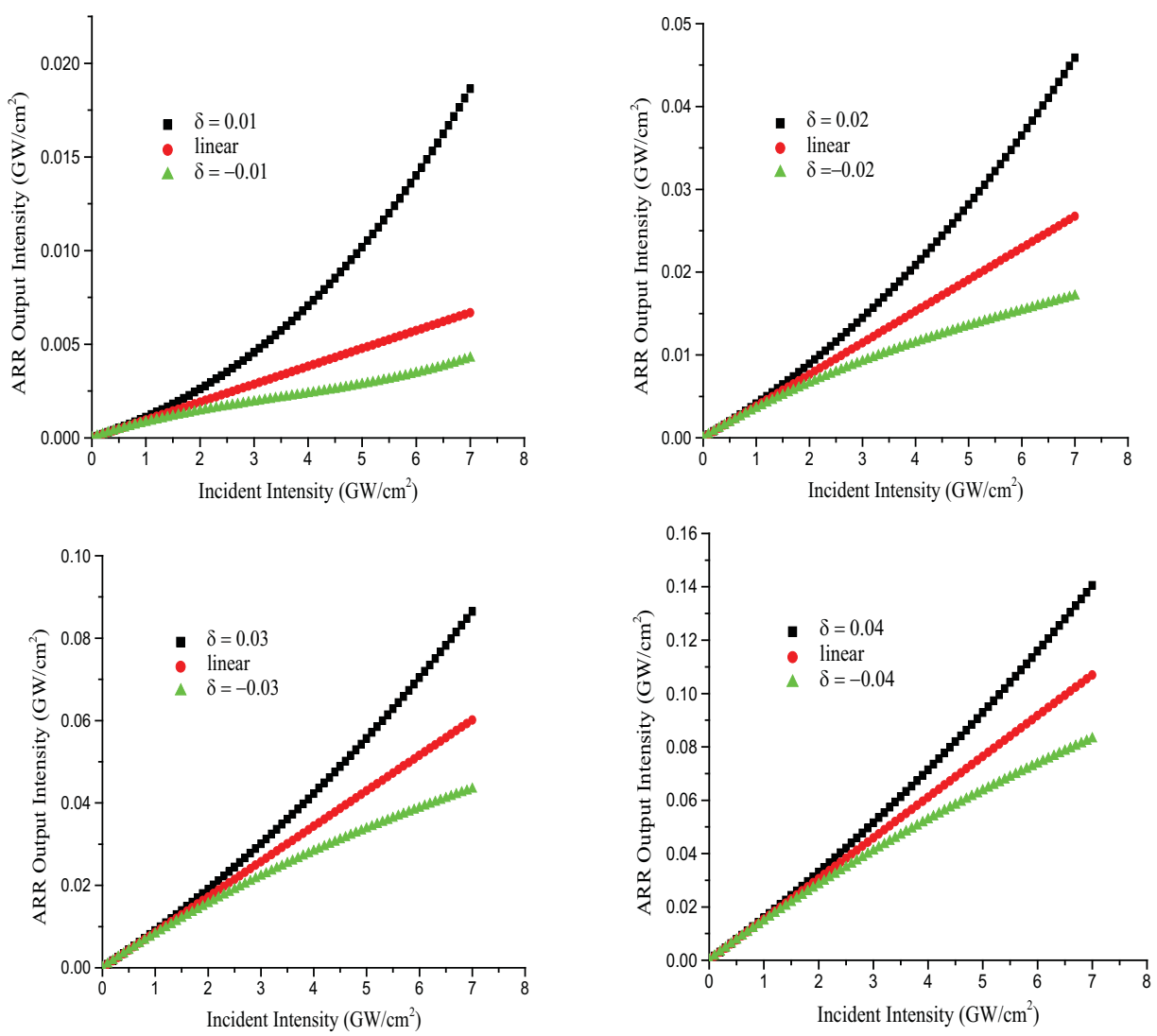

Fig. 18. ARINS simulation for (a) $\delta= \pm 0.01$, (b) $\delta= \pm 0.02$, (c) $\delta= \pm 0.03$ and (d) $\delta= \pm 0.04$ 


\section{Z-Scan and ARINS: a comparative discussion}

Since Z-Scan is a simple single-beam technique, it has no difficult alignment other than keeping the beam centered on the aperture. It yields magnitude as well as sign of $\chi^{(3)}$. It is also possible to separate out refractive and absorptive parts (real and imaginary parts) of the nonlinearity by performing simultaneous closed and open aperture scans. However, it gets affected by external noise. Since the sample passes through the beam waist, catastrophic self-focusing can damage the sample, particularly in thin films if significant absorption is present. Since the whole principle is based on the wavefront modification due to nonlinear effect, anomalies like sample scatter and surface inhomogeneity can cause artifact to Z-scan signatures.

On the contrary, both beams in ARINS travel the same path inside the ring and thus, any external jerk will affect both beams identically [71]. Also ARINS is very sensitive and can detect changes of less than $0.005 \%$ in the laser amplitude. Hence it can be used to measure nonlinearity of thin films having thickness as low as a few tens of nm. Furthermore, this technique has the unique capability of discriminating against different nonlinear optical processes based on their response times. Any value of $\delta$ results in a leakage from the ARR and is responsible for the background that limits the sensitivity of the measurement. Therefore, the alignment of the technique has to be very precise so as to keep $\delta$ as low as possible $(\sim 0.02)$. This technique does not provide the sign of nonlinearity. It has to be supplemented by other technique like Z-scan to get the sign of $\beta$ and $n_{2}$. Since ARINS technique is based on the time difference between the arrivals of two pulses at the sample, it cannot be used for cw beam.

Keeping in view of the potential advantages of Z-Scan and ARINS techniques over each other, a combination of these two techniques has recently been applied successfully to have very accurate estimates of the third-order optical susceptibilities of ion beam synthesized metal (copper, gold, silver) nanocluster glass composites [72, 73]. The silver ion implanted samples have shown the highest nonlinear susceptibility of $\sim 10-10$ e.s.u at off-resonant condition and $\sim 10^{-6}$ e.s.u at resonant condition, i.e at wavelength near to SPR of silver nanoparticles. The gold and copper implanted samples exhibited nonlinearty of the order $10^{-12}$ e.s.u. Attempts towards achieving higher orders of $\chi^{(3)}$ of such nanocomposites in the femtosecond time domains are, however, of prime interest for futuristic device technology.

\section{Photonic applications: scope and challenges}

Nonlinear optics has stirred many phenomena like fabrication of metal nanocluster-based novel nonlinear materials, harmonic generations, optical solitons, parametric amplification, stimulated Raman scattering, self-induced transparency, modulational instability, etc., which find a variety of applications ranging from high data transmission in optical communication, switching, amplifiers, pulse reshaping, pulse compression, tunable lasers to encoded message transmission. Surface plasmon absorption of metal nanoparticles have found large applications for chemical and biological sensing due to sensitive spectral response to local surface environment and ease of monitoring the light signal due to strong light scattering and absorption [74]. Due to the enhanced third-order nonlinear 
susceptibility of the metal nanoclusters, especially near the SPR frequency, these nanoparticles embedded in dielectric matrices have practical applications in ultrafast alloptical switching devices [75-78]. All optical switches are the base for optical parallel data processing systems and optical time domain multiplexing transmission systems. Nonlinear optical switching devices are based on optical waveguide structures for they provide strong beam confinement in prescribed patterns. In these devices, the intensity of an optical signal is used as a parameter that causes switching between two output channels that perform logic operations.

Today, there are a large number of nonlinear optical materials for specific wavelengths, with various damage thresholds, and with various optical characteristics. The major areas of interest are photonic metamaterials (materials having negative refractive index) and plasmonics, which bring together the two scientific communities of nanoplasmonics and metamaterials, both of which deal with tailored metal/dielectric and metal/semiconductor nanostructures.

Recently, metal nanocluster glass composites displaying negative refractive index - a property not found in any known naturally occurring materials - have drawn significant scientific interest, underscoring the remarkable potential of metamaterials to facilitate new developments in electromagnetism. Sophisticated structures for such metamaterials have to be prepared by various design technologies. Lots of groups throughout the world are involved in the synthesis of these kinds of materials. Different approaches have been made to synthesize the negative refractive index materials. These include fabricating a multilayer metal-dielectric stack by using focused ion-beam milling [79], printed circuit board technology [80], self assembly approach [81, 82], fabricating regular arrays of metal pillars by high-resolution electron-beam lithography on a glass substrate $[83,84]$, and by sol-gel technique [85]. Regular arrays of gold and silver pillars fabricated by high resolution electron-beam lithography or by ion-implantation, both on glass substrates have been reported to have negative refractive index. By providing access to new realms of material response, metal nanoclusters in glasses have and will continue to impact the fields of physics, materials science, engineering, optics, nanotechnology, and many other branches of science. Gold and silver are the materials of first choice for the realization of a large number of interesting nanoscale devices and structures due to their unique chemical and optical properties. Moreover, nanocomposites made of silver and gold nanowires imbedded in a sol-gel host have been morphologically and optically investigated.

Metallic nanoparticles have distinctly different optical characteristics than SPs at planar interfaces. Nanoparticles show strong optical resonances, again because of their large freeelectron density. As a result, a plane wave impinging on a $20 \mathrm{~nm}$ diameter $\mathrm{Ag}$ particle is strongly 'focused' into the particle, leading to a large electric field density in a $10 \mathrm{~nm}$ region around the particle. Ordered arrays of nanoparticles can possess even further enhanced field intensities as a result of plasmon coupling between adjacent particles [86]. By varying nanoparticle shape or geometry, the SP resonance frequency can be tuned over a broad spectral range. For example, Au ellipsoids or silica colloids covered with an Au shell show resonances that coincide with the important telecommunications wavelength band. The ability to achieve locally intense fields has many possible applications, including increasing the efficiency of LEDs, (bio-) sensing, and nanolithography.

Arrays of metal nanoparticles can also be used as miniature optical waveguides. In linear chain arrays of nanoparticles, a plasmon wave propagates by the successive interaction of 
particles along the chain. The propagation length is small $(\sim 100 \mathrm{~nm})$, but may be increased by optimizing particle size and anisotropy. What makes these nanoparticle array waveguides attractive is that they provide confinement of light within $\sim 50 \mathrm{~nm}$ along the direction of propagation, a 100-fold concentration compared to dielectric waveguides.

\section{References}

[1] P. A. Franken, A. E. Hill, C. W. Peters, and G. Weinreich, Phys. Rev. Lett. 7, 118 (1961)

[2] T. H. Maiman, R. H. Hoskins, I. J. D'Haenens, C. K. Asawa, and V. Evtuhov, Phys. Rev. 123 1151(1960)

[3] P. Chakraborty, Journal of Material Science, 332235 (1998)

[4] Robert W. Boyd, Nonlinear Optics, pp 4, Academic Press (2003)

[5] C.N.Ironside, Ultrafast all-optical Switching, Contemporary Physics, 34 (1993)

[6] G. Mie, Ann. Phys. (Leipz) 25, 377 (1908)

[7] M. Brack, Rev. Mod. Phys. 65, 677 (1993)

[8] C. Brechignac and J.P. Connerado, J. Phys. B : At. Mol. Opt. Phys. 27, 3795 (1994)

[9] F. Alasia, R.A. Broglia, H.E. Roman, L.I. Serra, G. Colo and J.M. Pacheco, J. Phys. B : At. Mol. Opt. Phys. 27, L663-L649 (1994)

[10] M. Madjet, C. Guet and W.R. Johnson, Phys. Rev. A 51, 1327 (1995)

[11] A.V. Korol and A.V. Solov'yov, J. Phys. B : At. Mol. Opt. Phys. 30,1105 (1996)

[12]W. Ekardt, Phys. Rev. B 33, 8803 (1986)

[13] W. Ekardt, Phys. Rev. B 36, 4483 (1987)

[14] L.G. Gerchikov, A.N. Ipatov, A.V. Solov'yov and W. Greiner, J. Phys. B : At. Mol. Opt. Phys. 31, 3065 (1998)

[15] Jean-Patirick Connerade and Andrey V Solov'yov, arXiv : physics/02020661v1 (27th Feb 2002)

[16] J.C. Maxwell-Garnett, Philos. Trans. R. Soc A 203, 385 (1904)

[17] G.W. Arnold, J.A. Bordes, J. Appl. Phys. 48,1488 (1977)

[18] W. C. Huang and J. T. Lue, Phys. Rev. B 49, 179 (1994)

[19] Idem, ibid. 42414 (1965)

[20] W. J. Doyle, Phys. Rev. 111 1067(1958)

[21] M. Sheik-Bahae, M.Hutchings, D.C. Hagan, Dispersion of bound electronic nonlinear refracting solids, IEEE J. Quant. Elect. 1296

[22] Y.Gao, X.Zhang, Y.Li, H. Liu, Y.Wang, Q.Chang, W.Jiao and Y. Song, Opics Communications 251, 429, (2005)

[23] G. Battaglin, R. Polloni, G. De Marchi, F. Cavale, F. Gonella, G. Mattei, P. Mazzoldi, A. Quaranta, F. Spizzo, G. De and R.F. Haglund Jr, in 'Proceedings of the International Conference on Fibre Optics and Photonics, Photonics -96' (Ed: J.P. Raina and P.R. Vaya), Tata McGraw-Hill Publishing Co., New Delhi, pp-36

[24] M. Mennig, M. Schmitt and H. Schmidt, J. Sol-Gel Sci. Tech. 8, 1035 (1997)

[25] G.W. Arnold, J. Appl. Phys. 46, 4466 (1975)

[26] F. Gonella, P. Mazzoldi, in Handbook of Nanostructured Materials and Nanotechnology, Vol. 4 (Ed: Hari Singh Nalwa) pp 81-158, Academic Press (2000).

[27] E. Cattaruzza, Nucl. Instr. and Meth. B 169, 141 (2000)

[28] G. Mattei, Nucl. Instr. and Meth. B 191, 323 (2002)

[29] F. Gonella, Nucl. Instr. and Meth. B 166, 831(2000) 
[30] R.F. Haglund, Jr., L. Yang, R.H. Magruder III, C.W. White, R.A. Zuhr, L.Yang, R. Dorsinville and R.R. Alfano, Nucl. Instr. and Meth. B 91, 493 (1994)

[31] N. Skelland and P.D. Townsend, Nucl. Instr. and Meth. B 93, 433 (1994)

[32] A. Meldrum, R.F. Haglund, L.A. Boatner and C.W. White, Adv. Mater. 13, 1431 (2001)

[33] F. Garrido, F. Caccavale, F. Gonella, A. Quaranta, J. Eur. Opt. Soc. A: Pure Appl. Opt. 4, $771(1995)$

[34] F. Garrido, J.C. Dran, L. Thome, C. Meneghini, F. Gonella and A. Quaranta Nucl. Instr. and Meth B 115, 561 (1996)

[35] F. Gonella, G. Mattei, P. Mazzoldi, C. Sada, G. Battaglin and E. Cattaruzza, Appl. Phys. Lett. 75, 55 (1999)

[36] D. Ila, Z. Wu, C.C. Smith, D.B. Poker, D.K. Hensley, C. Klatt, S. Kalbitzer, Nucl. Instr. and Meth B 127/128, 570 (1997)

[37] B. Ghosh, P. Chakraborty, B. Sunderavel and C. Vijayan, Nucl. Instr.and Meth. B 266, 1356 (2008)

[38] H. Hosono, Jpn. H. Hosono. Jpn. J. Appl. Phys. 32, 3892 (1993)

[39] H. Hosono and N. Matsunami. Phys. Rev. B 48, 13469 (1993)

[40] H. Hosono and H. Imagawa. Nucl. Instr. and Meth. B 91, 510 (1994)

[41] R. H. Magruder III, J. E. Witting and R. A. Zuhr, J. Non-Cryst. Solids 163, 162 (1993).

[42] P. D. Townsend, Rep. Prog. Phys. 50, 501 (1987).

[43] K. Uchida, S. Kaneko, S. Omi, C. Hata, H. Tanji, Y. Asahara, A. J. Ikushima, T. Tokizaki, and A. Nakamura, J. Opt. Soc. Am. B, Opt. Phys., 11 (7), 1236 (1994)

[44] R. Philip, G. Ravindra Kumar, N. Sandhyarani, and T. Pradeep, Phys. Rev. B, Condens. Matter, 62 (19), 13160 (2000)

[45] M. Falconieri, G. Salvetti, E. Cattaruzza, F. Gonella, G Mattei, P. Mazzoldi, M. Piovesan, G. Battaglin, and R. Polloni, Appl. Phys. Lett., 73 (3), 288 (1998)

[46] R.H. Magruder, III, R.F. Haglund,Jr. , L. Yang, J.E. Witting and R.A. Zuhr, J. Appl. Phys. 76, 708 (1994)

[47] R.L. Zimmerman, D. Ila, E.K. Williams, B. Gasic, A. Elsamadicy, A.L. Evelyn, D.B. Poker, D.K. Hensley, David J. Larkin, , Nucl. Instr. and Meth. B 166-167, 892 (2000)

[48] G. Battaglin, E. Cattaruzza, F. Gonella, G. Mattei, P. Mazzoldi, C. Sada and X. Zhang, Nucl. Instr. and Meth. B 166, 857 (2000)

[49] L. C. Nistor, J. Van Landuyt, J. D. Barton, D.E. Hole, N.D. Skelland and P.D. Townsend, J. Non-Cryst. Solids 162, 217 (1993)

[50] D. Ricard, P. Roussognal and C. Flytzanis, Opt. Lett. 10, 511 (1985)

[51] M. Sheik-Bahae, A. A. Said, T. Wei, D.J. Hagan and E.W. Van Stryland, IEEE J. Quantum Eletcron. QE-26, 760 (1990)

[52] R. F. Haglund, Jr., L. Yang, R.H. Magruder III, J.E. Witting, K. Becker and R.A. Zuhr, Opt. Lett, 18, 373 (1993)

[53] P. Mazzoldi, F. Caccavale, E. Cattaruzza, P. Chakraborty, L. Tramontin, A. BoscoloBoscoletto, R. Bertoncello, E. Trivillin, G. Battaglin and G. W. Arnold, Nucl. Instr. and Meth. B 91, 505 (1994)

[54] P. Mazzoldi, T. Tramontin, A. Boscolo-Boscoletto, G. Battaglin and G. W. Arnold, ibid. 80/81, 1192 (1993) 
[55] R. H. Magruder III, Do Henderson, S.H. Morgan and A. Zuhr. J. Non-Cryst. Solids 152, $258(1993)$

[56] H. Hosono, Y. Y. Suzuku, Y. Abe, Y. L. Lee, K. Oyoshi and S. Tanakar, ibid. 142, 287 (1992)

[57] Y. Takeda, T. Hoiki, T. Motohiro and S. Noda, Nucl. Instr. and Meth. B 91, 505 (1987)

[58] D. Ila, E.K. Williams, S. Sarkisov, C.C. Smith, D.B. Poker and D.K. Hensley, Nucl. Instr. and Meth B 141, 289 (1998)

[59] Y. Menke, M. Ferraris, C. Corbari and J. Fage-Pedersen, Journal of Non-Crystallien Solids, 345/346, 366 (2004)

[60] H. H. Huang, F. Q. Yan, Y. M. Kek, C. H. Chew, G. Q. Xu, W. Ji, P. S. Oh and S. H. Tang, Langmuir, 13(2), 172 (1997)

[61] Y. H. Wang, Y.M. Wang, J.D. Lu, L.L. Ji, R.G. Zang and R.W. Wang, Optics Communications 283(3), 486 (2010)

[62] B. Ghosh, P. Chakraborty, C. Vijayan, S. Mahapatra and P. Mazzoldi, Materials Letters 61, 4512(2007)

[63] P. Prem Kiran, G. De and D. Narayana Rao, IEE Proc.-Circuits Devices Syst. 150(6), 559 (2003)

[64] R. H. Magruder III, J. E. Witting and R. A. Zuhr, J. Non-Cryst. Solids 163, 162 (1993).

[65] R. C. Caro and R. C. Gower, IEEE J. Quantum Electron. QE-18, 1375 (1982)

[66] R. H. Magruder III, L. Yang, R. F. Haglund, C. W. White, Lina Yang, R. Dorsinville and R. R. Alfano, Appl. Phys. ,Lett. 621730 (1993).

[67] Y. R. Shen, The principles of Nonlinear Optics, Wiley, pp 1-563, New York, (1984)

[68] H. W. H. Lee, R.S. Hughes, Opt. Lett. 19, 1708 (1994)

[69] Parinda Vasa, B.P.Singh, Praveen Taneja, Pushan Ayub, Optics Communications 233, 297 (2007).

[70] M. C. Gabriel, N.A. Whitaker Jr., C.W. Dirk, M.G.Kuzyk and M. Thakur, Opt. Lett. 16 1334 (1991)

[71] R. Trebino, C. C. Hayden, Opt. Lett. 16, 493(1991)

[72] B. Ghosh and P. Chakraborty, Nucl. Instrum. Meth B (in press), doi: 10.1016/j.nimb 2010.11.055 (2010).

[73] B. Ghosh, P. Chakraborty, B. P. Singh and T. Kundu, Applied Surface Science, 256, 389 (2009)

[74] Y. Xia, N.J. Halas, MRS Bulletin, 30, 338 (2005)

[75] N. Pincon, B. Palpant, D. Prot, E. Charron, S. Debrus, Eur. Phys. J. D 19, 395 (2002)

[76] H. S. Jun, K. S. Lee, S. H. Yoon, T. S. Lee, I.H. Kim, J. H. Jeong, B. Cheong, D. S. Kim, K. M. Cho, W.M. Kim, Phys. Stat. Sol. (A), 203, 1211 (2006)

[77] M. S. Hu, H. L. Chen, C. H. Shen, L. S. Hong, B. R. Huang, K. H. Chen, L. C. Chen, Nat. Mater. 5, 102 (2006)

[78] W. Schrof, S. Rozouvan, E.V. Keuren, J. Schmitt, G. Decher, Adv. Mater. 3, 338 (2002)

[79] Jason Valentine et al, NATURE LETTERS 455, 376 (2008)

[80] Dongo Kim, Wangju Lee and Jaeick Choi, Appl Phys A 97, 461 (2009)

[81] J. F Galisteo et. al , Journal of Optics A: Pure Appl. Opt. 7, 244 (2005)

[82] Santhi Ani Joseph et al, Journal of Crystal Growth 311, 1152 (2009) 
[83] A. N. Grigorenko et. al, Appl. Phys. Lett. 88, 124103 (2006)

[84] A. Perentes and I. Utke, Nanotechnology 16, 273 (2005)

[85] A. M. Chinie, A. Stefan and S. Georgescu, Romanian Reports in Physics 57, 412 (2005)

[86] Albert Polman and Harry A. Atwater, Materials Today, January 2005, p. 56 


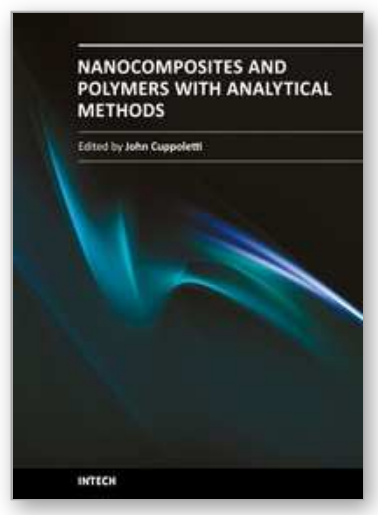

\author{
Nanocomposites and Polymers with Analytical Methods \\ Edited by Dr. John Cuppoletti
}

ISBN 978-953-307-352-1

Hard cover, 404 pages

Publisher InTech

Published online 09, August, 2011

Published in print edition August, 2011

This book contains 16 chapters. In the first part, there are 8 chapters describing new materials and analytic methods. These materials include chapters on gold nanoparticles and Sol-Gel metal oxides, nanocomposites with carbon nanotubes, methods of evaluation by depth sensing, and other methods. The second part contains 3 chapters featuring new materials with unique properties including optical non-linearities, new materials based on pulp fibers, and the properties of nano-filled polymers. The last part contains 5 chapters with applications of new materials for medical devices, anodes for lithium batteries, electroceramics, phase change materials and matrix active nanoparticles.

\title{
How to reference
}

In order to correctly reference this scholarly work, feel free to copy and paste the following:

Binita Ghosh and Purushottam Chakraborty (2011). Optical Nonlinearities of Colloidal Metal Quantum Dot Glass Composites for Nanophotonics, Nanocomposites and Polymers with Analytical Methods, Dr. John Cuppoletti (Ed.), ISBN: 978-953-307-352-1, InTech, Available from:

http://www.intechopen.com/books/nanocomposites-and-polymers-with-analytical-methods/opticalnonlinearities-of-colloidal-metal-quantum-dot-glass-composites-for-nanophotonics

\section{INTECH}

open science | open minds

\section{InTech Europe}

University Campus STeP Ri

Slavka Krautzeka 83/A

51000 Rijeka, Croatia

Phone: +385 (51) 770447

Fax: +385 (51) 686166

www.intechopen.com

\section{InTech China}

Unit 405, Office Block, Hotel Equatorial Shanghai

No.65, Yan An Road (West), Shanghai, 200040, China

中国上海市延安西路65号上海国际贵都大饭店办公楼 405 单元

Phone: $+86-21-62489820$

Fax: $+86-21-62489821$ 
(C) 2011 The Author(s). Licensee IntechOpen. This chapter is distributed under the terms of the Creative Commons Attribution-NonCommercialShareAlike-3.0 License, which permits use, distribution and reproduction for non-commercial purposes, provided the original is properly cited and derivative works building on this content are distributed under the same license. 